\title{
Guiding ATR and PARP inhibitor combinations with
}

\section{chemogenomic screens}

Michal Zimmermann ${ }^{1, *}$, Cynthia Bernier ${ }^{1}$, Beatrice Kaiser ${ }^{1}$, Sara Fournier ${ }^{1}$, $\mathrm{Li} \mathrm{Li}^{1}$, Jessica Desjardins ${ }^{1}$, Alexander Skeldon², Victoria Rimkunas ${ }^{3}$, Artur Veloso ${ }^{3}$, Jordan T. F. Young ${ }^{1}$, Anne Roulston ${ }^{1}$, Michael Zinda ${ }^{3}$

${ }^{1}$ Repare Therapeutics, 7171 Rue Frederick-Banting, St-Laurent, QC H4S 1Z9, Canada

${ }^{2}$ Ventus Therapeutics, 7150 Rue Frederick-Banting, St-Laurent, QC H4S 2A1, Canada

${ }^{3}$ Repare Therapeutics, 1 Broadway, 15th Floor, Cambridge, MA 02142, USA

${ }^{*}$ Correspondence should be addressed to: mzimmermann@reparerx.com

Key words: ATR inhibitor, PARP inhibitor, Drug Combination, CRISPR screen, Synthetic Lethality

Character count: 44,996 (excluding Summary, Materials and Methods, References and Supplementary Figure legends)

Number of figures: 6

Number of references: 76

Supplementary Material: 8 figures, 1 table 


\section{SUMMARY (150 words)}

2 Combinations of inhibitors of Ataxia Telangiectasia- and Rad3-related kinase (ATRi)

3 and poly(ADP-ribose) polymerases (PARPi) synergistically kill tumor cells through

4 modulation of complementary DNA repair pathways, but their tolerability is limited by

5 hematological toxicities. To address this we performed a genome-wide CRISPR/Cas9

6 screen to identify genetic alterations that hypersensitize cells to a combination of the

7 ATRi RP-3500 with PARPi, including deficiency in RNase H2, RAD51 paralog mutations

8 or the Alternative Lengthening of Telomeres telomere maintenance mechanism. We

9 show that RP-3500 and PARPi combinations kill cells carrying these genetic alterations

10 at doses sub-therapeutic as single agents. We also demonstrate the mechanism of

11 combination hypersensitivity in RNase $\mathrm{H} 2$-deficient cells, where we observe an

12 irreversible replication catastrophe, allowing us to design a highly efficacious and

13 tolerable in vivo dosing schedule. Altogether, we present a comprehensive dataset to

14 inform development of ATRi and PARPi combinations and an experimental framework

15 applicable to other drug combination strategies. 


\section{INTRODUCTION}

17 Rational combinations of cytotoxic chemotherapeutics have been long used in cancer

18 treatment as they can synergistically enhance anti-tumor activity compared to single

19 agent regimens and may delay or overcome the emergence of drug resistance (Frei et

20 al., 1965). The recent clinical development of cancer therapies based on synthetic lethal

21 targeting of the DNA damage response (DDR) pathways provides an exciting

22 opportunity for the design of novel drug combinations, since most types of DNA damage

23 can be repaired by parallel pathways and complementary mechanisms of action. DDR

24 targeting compounds are thus prime candidates for eliciting synergistic activity when

25 used in combination (Brown et al., 2017; Pilié et al., 2019; Setton et al., 2021).

26 However, drug combination synergism is often accompanied by increased systemic

27 toxicity, and strategies must be developed to improve tolerability, for example by

28 rational patient selection and/or careful dose-scheduling (Brown et al., 2017; Fang et al.,

29 2019; Pilié et al., 2019).

The first application of synthetic lethality in cancer therapy was the use of

31 poly(ADP-ribose) polymerase inhibitors (PARPi) against BRCA1/2-deficient tumors

32 (Bryant et al., 2005; Farmer et al., 2005). PARPi monotherapy has been approved in

33 several clinical settings since the initial discovery of its synthetic lethal potential

34 (Hussain et al., 2020; Pujade-Lauraine et al., 2017; Robson et al., 2017) and multiple

35 PARPi combinations are currently under investigation (Brown et al., 2017; Pilié et al.,

36 2019). Another class of compounds targeting the DDR based on synthetic lethality are

37 inhibitors of Ataxia Telangiectasia- and Rad3-related (ATR) kinase, a key regulator of

38 the replication stress response (Bradbury et al., 2020; Lecona and Fernandez-Capetillo, 
2018). Multiple ATR inhibitors (ATRi) are in clinical development as monotherapies and

40 in combination with PARPi, including RP-3500, a novel, highly potent, selective, and

41 orally bioavailable ATRi that has shown robust preclinical efficacy in models deficient in

$42 B R C A 1 / 2$ or the Ataxia Telangiectasia-Mutated kinase gene (ATM) (Roulston et al.,

432021 , manuscript in press).

Combining PARPi and ATRi is based on a strong mechanistic rationale, as

45 PARPi create DNA damage that engages the ATR pathway to facilitate cell survival.

46 After activation by replication-associated single-stranded DNA (ssDNA) lesions, ATR

47 coordinates the DDR in multiple ways: First, ATR activates a cell cycle checkpoint to

48 prevent mitosis before DNA damage is repaired. Second, ATR blocks firing of dormant

49 replication origins to avoid further propagation of replication stress. Lastly, ATR

50 mediates stabilization and repair/restart of stalled replication forks by activating fork

51 remodeling enzymes and DNA repair by homologous recombination (reviewed by

52 Saldivar et al., 2017). PARPi create S-phase DNA damage by preventing the release of

53 PARP enzymes from their DNA-bound state, a phenomenon known as 'PARP trapping'

54 (Murai et al., 2012), as well as by other mechanisms, e.g., preventing repair of ssDNA

55 replication gaps (Cong et al., 2021). In BRCA1/2-deficient cells, ATRi prevent cellular

56 recovery from PARPi treatment and cause rapid cell death by premature mitotic entry

57 with unrepaired DNA damage and inflammatory signaling (Bryant et al., 2005; Farmer et

58 al., 2005; Kim et al., 2017; Schoonen et al., 2019). Consequently, combinations of ATRi

59 and PARPi show synergistic cytotoxicity in cell viability assays and enhanced in vivo

60 efficacy over each single agent in pre-clinical BRCA1/2- or ATM-deficient tumor models

61 (Kim et al., 2020, 2017; Lloyd et al., 2020). Furthermore, in a Phase I clinical study, a 
62 combination of the ATRi AZD6738 with the PARPi olaparib showed signals of activity in

63 BRCA1/2-mutated tumors (Yap et al., 2016). ATRi have also been shown to overcome

64 several mechanisms of acquired PARPi resistance in preclinical models (Kim et al.,

65 2020; Murai et al., 2016; Yazinski et al., 2017), suggesting a potential application in a

66 PARPi-resistant tumor setting.

67 Despite the proven synergy, taking full advantage of ATRi/PARPi combinations

68 has been hindered by their limited tolerability due to hematological toxicities (Fang et

69 al., 2019; Yap et al., 2016). Pre-clinical proof-of-concept studies showed that this

70 problem could be overcome by the identification of tumors carrying hypersensitizing

71 alterations, such as BRCA1/2 or ATM-deficiency, which would allow the use of lower

72 doses while maintaining efficacy (Kim et al., 2017; Lloyd et al., 2020). However, a

73 comprehensive genome-wide map of genetic alterations that cause hypersensitivity to

74 ATRi/PARPi combinations is not available. Another approach to mitigate the systemic

75 toxicity is the design of novel dose schedules to minimize the effect of the combination

76 on normal dividing tissues (Fang et al., 2019). Successful clinical development of

77 ATRi/PARPi combinations will likely require application of these two approaches in a

78 concerted fashion.

In recent years, CRISPR/Cas9 chemogenomic screening has proven useful in

80 mapping cellular responses to many compounds used in cancer therapy, including

81 PARPi and ATRi (Hustedt et al., 2019; Olivieri et al., 2020; Wang et al., 2019;

82 Zimmermann et al., 2018). In case of ATRi, a 'consensus' set of sensitizing alterations

83 has been proposed by compiling multiple parallel CRISPR screening datasets (Hustedt

84 et al., 2019). Here we employ this technology to chart the cellular factors mediating the 
85 response to a ATRi/PARPi combination and identify cancer-relevant genetic alterations

86 that cause profound sensitization. Furthermore, we describe the mechanism by which

87 cells carrying one of these alterations, loss of the RNase $\mathrm{H} 2$ enzyme, are affected by

88 the combination and use this information to design an optimized, efficacious in vivo

89 dosing schedule that is well tolerated in mouse models. Altogether, we present an

90 experimental framework that can be adapted to many drug combination strategies and

91 show that this framework generates novel insights into the use of ATRi/PARPi

92 combinations that can inform clinical development. 


\section{RESULTS}

95 A chemogenomic screen for genes underlying the cellular response to single agent and combination treatment with ATR and PARP inhibitors

97 To map the cellular response to RP-3500 alone or in combination with PARPi we

98 performed a CRISPR chemogenomic screen in Cas9-expressing RPE1-hTERT TP53

99 knock out (KO) cells using the TKOv3 library (Hart et al., 2017; Olivieri and Durocher, 100 2021). We treated cells with dimethyl sulfoxide (DMSO), the ATRi RP-3500, the PARPi

101 niraparib or both in combination (Figure 1A). We analyzed data using the

102 CRISPRCountAnalysis (CCA) algorithm (Adam et al., 2021), which stratifies statistically 103 significant drug sensitizer hits into four 'Jenks classes', with Jenks class 4 being the 104 strongest hits and Jenks class 1 being the weakest (Supplementary Table 1). We 105 identified 122 gene hits in Jenks classes 3 and 4, whose targeting single guide RNA 106 (sgRNA) led to sensitivity to either RP-3500 alone, niraparib alone, or RP-

107 3500/niraparib. Among these genes, 12 scored as hits in all three treatment arms, 108 whereas several arm-specific hits were identified, including 18 genes specific to the

109 combination arm (Figure 1B, Supplementary Table 1). We will hereafter refer to these

110 genes as 'high-confidence' hits. When we extended the analysis to all Jenks classes,

111 we obtained a total of 377 hits, of which 46 scored in all arms and 79 were specific to

112 the combination (Supplementary Figure 1A, Supplementary Table 1). We will refer to 113 this dataset as the 'extended' hit list. 
115 Robust detection of known DNA repair factors following single agent ATR and

116 PARP inhibitor treatments

117 The cellular response to single agent ATRi and PARPi has been previously profiled

118 using chemogenomic screening (Bajrami et al., 2014; Hustedt et al., 2019; Olivieri et al.,

119 2020; Wang et al., 2019; Zimmermann et al., 2018) and we could therefore use this

120 information to 'benchmark' the ability of our CRISPR-enabled screening pipeline to

121 detect bona fide sensitizing gene alterations. We used PANTHER (Mi et al., 2021) to

122 determine in an unbiased way, which Gene Ontology (GO) biological processes were

123 enriched in each arm of our screen (Figure 1B). Both single agent ATRi and PARPi

124 arms readily identified homologous recombination (HR) and other genome stability-

125 related processes, confirming the screens' robustness. Of note, whereas the pathways

126 responding to PARPi treatment were (as expected) centered around HR, several

127 additional ATRi-specific pathways were identified (including translesion synthesis,

128 Figure 1B).

Next, we compared our single agent ATRi dataset to the published 'consensus'

130 set of ATRi-sensitizing genes from Hustedt et al. (Hustedt et al., 2019) and found 35

131 overlapping genes (Supplementary Figure 1B), including ATM, the three RNase H2

132 subunits RNASEH2A, $B$ and $C$, as well as several HR and DNA replication factors

133 (Supplementary Figure 1B, Supplementary Table 1). To validate RP-3500 sensitivity

134 after inactivation of selected hits (ATRIP, RAD17, REV3L, SETD2, CHTF8, FZR1), we

135 used small interfering RNA (siRNA) pools in two or three human cell lines (RPE1-

136 hTERT Cas9 TP53-KO, MCF10A and HeLa). Knockdown of each gene sensitized at

137 least one cell line to RP-3500 to a comparable, or greater, extent than knockdown of 
138 ATM (Supplementary Figure 1C). Collectively, these data confirm that our CRISPR-

139 enabled screening pipeline is robust and able to detect true sensitizers to genotoxic

140 perturbations.

142 Identification of genetic vulnerabilities to combined ATR and PARP inhibition

143 To identify cancer-relevant determinants of sensitivity to ATRi and PARPi combinations

144 we focused on genes scoring in all three treatment arms, as overlapping sensitivity to

145 each single agent should allow achievement of drug synergy at low doses. Among the

14612 high-confidence hits were 6 genes with known deleterious alterations in human

147 tumors (Cancer Genome Atlas Research Network et al., 2013): ATM, RNASEH2A,

148 RNASEH2B, RNASEH2C, and two RAD51 paralogs, RAD51B and RAD51D (Figure

149 1B, Supplementary Figure 1D, Supplementary Table 1). A strong vulnerability of

150 ATM-deficient tumor cells to combined ATRi and PARPi has been shown previously

151 (Lloyd et al., 2020). RNase $\mathrm{H} 2$ is a protein complex composed of three individual

152 polypeptides - the catalytic RNASEH2A subunit and two scaffolding subunits,

153 RNASEH2B and RNASEH2C, in a manner where all three proteins are essential for the

154 stability and activity of the complex (Reijns et al., 2011; Reijns and Jackson, 2014).

RNase $\mathrm{H} 2$ plays a key role in the ribonucleotide excision repair pathway, which is

156 responsible for removal of ribonucleotides mis-incorporated into DNA by replicative

157 polymerases (Pizzi et al., 2015; Reijns et al., 2012; Reijns and Jackson, 2014). Biallelic

158 loss of the RNASEH2B gene can be found in a subset ( 14\%) of chronic lymphocytic

159 leukemias as part of the 13q14 tumor suppressor locus deletion (which includes RB1 
160 and the $D L E U / m i R-15 a / m i R-16-1$ cluster) (Zimmermann et al., 2018). Possible

161 RNASEH2B loss in other tumor types carrying $R B 1$ homozygous deletions is being

162 investigated (Wang et al., 2019; Zimmermann et al., 2018). RAD51 paralogs play

163 important roles as chaperones of the RAD51 nucleofilament formation during HR and

164 maintenance of stalled replication forks (reviewed in Bonilla et al., 2020; Rein et al.,

165 2021). Consistent with this function, germline mutations in RAD51B, RAD51C and

$166 R A D 51 D$ predispose to breast or ovarian tumors and early PARPi efficacy signals in

167 RAD51 paralog-mutated tumors are emerging in the clinic (Akbari et al., 2010; Golmard

168 et al., 2013; Loveday et al., 2011; Swisher et al., 2021).

169 Of note, when mining our extended datasets, we found other factors associated

170 with the same pathways as the 12 high-confidence hits. NBS1/NBN, a member of the

171 MRE11-NBS1-RAD50 (MRN) complex required for ATM activation (Syed and Tainer,

172 2018) scored as a combination-specific hit (Supplementary Figure 1D,

173 Supplementary Table 1). Overlapping ATRi and PARPi sensitivities of MRE11-

174 deficient cells were shown previously (Fagan-Solis et al., 2020) and we demonstrated

175 efficacy of RP-3500 in a model carrying a MRE11 alteration (Roulston et al., submitted).

176 Whether the PARPi combination would further enhance efficacy in this model remains

177 to be tested. Unsurprisingly, we also detected numerous HR factors; however, we do

178 highlight that in addition to $R A D 51 B$ and $R A D 51 D$, we detected all remaining canonical

179 RAD51 paralogs, including RAD51C, XRCC2 and XRCC3 in our extended ATRi and

180 ATRi/PARPi datasets (Supplementary Figure 1D, Supplementary Table 1).

182 shown enhanced efficacy over single agent treatments (Kim et al., 2020, 2017; Lloyd et 
183 al., 2020). Therefore, next we evaluated whether this would apply also to the additional

184 genetic alterations identified here.

186 RNase H2-deficient cells are profoundly sensitive to combined ATR and PARP

187 inhibition

188 We and others have previously described single agent sensitivity of RNase H2-deficient 189 cells to PARPi and ATRi (Hustedt et al., 2019; Wang et al., 2019; Zimmermann et al., 190 2018). To validate the combination screen results, we examined whether RNASEH2B

191 KO cells had enhanced sensitivity to combined PARPi and ATRi compared to single

192 agent treatment and also their wild type (WT) isogenic counterparts using three

193 independent cellular backgrounds (5637, DLD1 and RPE1-hTERT TP53-KO)

194 (Zimmermann et al., 2018), Figure 2A,B, Supplementary Figure 2A). As expected, all

195 RNASEH2B-KO cells were sensitive to single agent RP-3500 and the PARPi niraparib

196 or talazoparib (Supplementary Figure 2B-K). Importantly, combining RP-3500 with

197 PARPi strongly enhanced this cytotoxic effect and doses, which were benign to WT

198 cells and sublethal in RNASEH2B-KO cells as single agents, were sufficient to nearly

199 eliminate RNASEH2B-KO cells (Figure 2C-E; Supplementary Figure 3A-D). We

200 determined that the cytotoxic effect of combined treatment was synergistic by utilizing

201 the SynergyFinder tool (lanevski et al., 2020; Yadav et al., 2015). Notably, in

202 RNASEH2B-KO cells concentrations of RP-3500 and PARPi that showed synergy were

203 markedly lower than in WT cells (Figure 2F-H; Supplementary Figure 3E). Together, 
204 these results validate that RNase H2-deficient cells are profoundly sensitive to

205 combinations of PARPi and ATRi.

206

207 Distinct response to PARPi and ATRi in cells lacking RAD51 paralogs

208 RAD51 paralogs are required for $\mathrm{HR}$ and it is not surprising that they were hits in our

209 screen. However, these proteins have additional and less explored roles in genome

210 stability maintenance, including replication fork protection and DNA damage tolerance,

211 and contributions of the different RAD51 paralogs to these non-HR functions remain

212 poorly understood (Rein et al., 2021). Interestingly, loss of individual RAD51 paralogs

213 has been shown to differentially impact PARPi sensitivity in vitro, whereby RAD51B

214 deficiency displays a milder phenotype than loss of other paralogs (Garcin et al., 2019).

215 As RAD51B was one of the strongest ATRi and ATRi/PARPi sensitizers in our screen,

216 we hypothesized that the response of RAD51 paralogs to ATRi or ATRi/PARPi may

217 differ from that of single agent PARPi. To test this, we depleted RAD51B, RAD51C or

218 RAD51D from RPE1-hTERT Cas9 TP53-KO cells with lentiviral sgRNAs and confirmed

219 that loss of RAD51C and RAD51D sensitized more strongly to the PARPi olaparib than

$220 R A D 51 B$, despite RAD51B sgRNAs displaying high cutting efficiency as judged by

221 Inference of CRISPR Edits (ICE) analysis (Supplementary Figure 4A; Note that

$222 s g R A D 51 C-2$ that causes lower olaparib sensitivity also induces a lower number of out-

223 of-frame indels; Hsiau et al., 2018). At the same time, the sensitivity to single agent RP-

2243500 was comparable between all three paralogs (Figure 3A-D). 
226 sensitized to a combination of RP-3500 and the PARPi olaparib (Figure 3E;

227 Supplementary Figure 4B). Doses of RP-3500/olaparib that were near indolent in

228 control (AAVS1-targeting) sgRNA-transduced cells substantially decreased viability of

229 cells transduced with sgRNAs targeting RAD51B, RAD51C or RAD51D, which was

230 accompanied by strong synergy between RP-3500 and olaparib occurring at lower

231 doses than in sgAAVS1-transduced cells (Figure 3E,F; Supplementary Figure 4B).

232 These data suggest that ATRi or ATRi/PARPi combinations may be universally effective

233 against RAD51 paralog-mutated tumor cells, unlike single agent PARPi. In contrast,

234 preclinical data suggest that $R A D 51 B$ alterations may reduce PARPi sensitivity

235 compared with alterations in RAD51C and RAD51D (Garcin et al., 2019), and the use of

236 ATRi or ATRi/PARPi combinations may overcome this issue.

Tumor cells utilizing alternative lengthening of telomeres are hypersensitive to ATRi/PARPi combinations

240 In search of other cancer-relevant genetic alterations sensitive to ATRi/PARPi

241 combinations, we noticed that the hits in our ATRi/PARPi screen arm were enriched for

242 several telomere-related GO processes (Figure 1B). Closer examination of the

243 combination dataset revealed the presence of several genes implicated in DNA repair

244 reactions at telomeres in cells utilizing the Alternative Lengthening of Telomeres (ALT)

245 pathway (Zhang and Zou, 2020). FAAP24, a member of the FANCM DNA translocase

246 complex, was a hit in the high-confidence combination dataset and FANCM itself scored

247 in the extended list (Supplementary Figure 5A, Supplementary Table 1). Moreover, 
248 the replication fork remodeling enzyme SMARCAL1 was a strong hit in the ATRi and 249 ATRi/PARPi screens (Supplementary Figure 5A, Supplementary Table 1). Both the 250 FANCM complex and SMARCAL1 have been shown to play key roles at ALT telomeres 251 and are known to be regulated by ATR signaling (Bansbach et al., 2009; Lu et al., 2019; 252 Poole et al., 2015; Silva et al., 2019; Singh et al., 2013). Of note, ATRX, a chromatin 253 remodeler whose inactivation is strongly associated with ALT (Zhang and Zou, 2020) 254 scored as a combination-specific hit (Supplementary Table 1), albeit we were unable 255 to confidently validate this gene using isogenic cell line pairs (data not shown).

256 Nevertheless, due to the presence of multiple ALT-associated factors in our dataset and 257 as ALT has been previously linked to single agent ATRi and PARPi sensitivity (Flynn et 258 al., 2015; Garbarino et al., 2021), we tested whether ALT-positive cancer cells can be 259 targeted with ATRi/PARPi combinations.

We assembled a panel of five ALT-positive (ALT+; U2OS, SAOS2, HS729T, 261 CAL72 and TM31) and five telomerase-positive (TEL+; HT1080, SJSA1, HS683, HOS 262 and HELA) cancer cell lines, and confirmed ALT-status using the recently developed 263 ssTeloC native fluorescence in-situ hybridization (FISH) assay that reflects the level of 264 extrachromosomal C-circles, a hallmark of ALT (Figure 4A,B; (Loe et al., 2020)). We 265 also included three non-cancerous telomerase-positive cell lines (RPE1-hTERT, COL266 hTERT and MCF10A) and performed cell viability assays with RP-3500, talazoparib, or 267 the combination. In agreement with previous results (Flynn et al., 2015) we observed 268 that ALT+ cell lines were on average more sensitive to ATR inhibition with single agent 269 RP-3500 than the TEL+ cell lines (Supplementary Figure 5B). The addition of 270 talazoparib exacerbated the cytotoxic effect of RP-3500, leading to near full loss of 
271 viability in ALT+ cell lines at doses that induced a considerably milder effect in TEL+

272 cancer cell lines and normal immortalized cell lines (Figure 4C). One exception was the

273 SAOS2 cell line, which was more resistant to ATRi/PARPi than other ALT+ cell lines as

274 well as TEL+ cells (Figure 4C). The reason for this discrepancy is unknown, but it may

275 point to redundancies or heterogeneity among ALT-related mechanisms (Verma et al.,

276 2019; Zhang et al., 2019)

277 To exclude the possibility that the ALT+ cell lines in our panel were sensitive to

278 RP-3500+PARPi for reasons unrelated to the ALT phenotype, we evaluated whether

279 ATRi/PARPi combinations affect ALT activity directly. Consistent with that possibility, we

280 observed an increase in ALT activity as measured by ssTeloC in the U2OS ALT+ cell

281 line upon RP-3500 treatment and a further synergistic increase in combination with

282 talazoparib (Figure 4D). Interestingly, this effect was not observed in SAOS2 cells,

283 which are resistant to the combination (Figure 4D). These results suggest that the

284 sensitivity to the combined ATRi/PARPi correlates with its impact on ALT activity.

285 Excessive ALT activity has been shown to be detrimental due to overwhelming levels of

286 telomere replication stress, as has recently been shown upon inactivation of FANCM

287 (Lu et al., 2019; Silva et al., 2019).

\section{A mechanism of ATRi/PARPi sensitivity in RNase H2-deficient cells}

289 Having identified multiple genetic alterations sensitizing to ATRi/PARPi combination, we 290 next sought to design an optimal dosing schedule for in vivo use of RP-3500/PARPi. To

291 achieve this, we first developed a deeper understanding of the mechanism by which the 292 combination affects sensitive genetic backgrounds. We focused on RNase $\mathrm{H} 2$ due to 
293 the profound sensitivity to the combination in cells mutated for RNase H2-encoding

294 genes. Furthermore, it was unclear how RNase H2-deficient cells are affected by

295 ATRi/PARPi combination in comparison to other alterations such as ATM or BRCA1/2

296 deficiency (Kim et al., 2017; Lloyd et al., 2020; Schoonen et al., 2019).

We initially monitored cell cycle progression and DNA damage accumulation

298 after PARPi, ATRi and ATRi/PARPi treatment using high-content microscopy in 5637

299 WT and RNASEH2B-KO cells with $\gamma$-H2AX as a marker of DNA damage, 4',6-diamidino-

300 2-phenylindole (DAPI) as a measure of DNA content and 5-ethynyl-2'-deoxyuridine

301 (EdU) to label cells replicating their DNA (Supplementary Figure 6A). The RP-

$3023500+$ niraparib combination led to marked DNA damage accumulation in RNASEH2B-

303 KO cells characterized by the appearance of pan-nuclear $\gamma$-H2AX (Figure 5A,B;

304 Supplementary Figure 6A-C). A substantial portion of pan- $\gamma$-H2AX-positive cells

305 showed >2N DNA content but did not incorporate EdU (Supplementary Figure 6A,B),

306 suggesting that these cells entered S-phase but are unable to complete DNA

307 replication. Consistent with defective DNA replication, we observed a marked decrease

308 in overall percentage of EdU-positive (EdU+) cells in the combination-treated

309 RNASEH2B-KO cells (Figure 5C; Supplementary Figure 6). At the same doses, both

310 compounds showed a much milder effect as single agents in RNASEH2B-KO cells and

311 no effect was observed in WT cells (even with the combination; Figure 5A-C;

312 Supplementary Figure 6). The DNA damage and replication defects observed in

313 RNASEH2B-KO cells treated with the RP-3500/niraparib combination were irreversible,

314 as washout did not lower $\gamma$-H2AX levels or restore defective EdU incorporation (Figure

315 5A-C; Supplementary Figure 6). 
317 arrest was reminiscent of the previously described phenotype of replication catastrophe

318 (RC) due to replication protein A (RPA) exhaustion (Toledo et al., 2013). To determine

319 whether the ATRi/PARPi combination induces RC in RNase H2-deficient cells, we

320 monitored the levels of chromatin-bound RPA together with $\gamma-\mathrm{H} 2 \mathrm{AX}$ (Toledo et al.,

321 2013). RP-3500+niraparib in both 5637 and RPE1 RNASEH2B-KO cells progressively

322 increased the RPA signal accompanied by appearance of high $\gamma-\mathrm{H} 2 \mathrm{AX}$ at later time

323 points, confirming RPA exhaustion and RC (Figure 5D-F, Supplementary Figure 7A-

324 C) (Toledo et al., 2013). Consistent with our prior results, we observed a near-total

325 absence of RC in 5637 WT cells following ATRi/PARPi and the effect of the combination

326 was two-fold greater than that of RP-3500 alone in RNASEH2B-KO cells (Figure 5D-F,

327 Supplementary Figure 7A-C).

$\mathrm{RC}$ is an irreversible process leading to cell death (Toledo et al., 2013),

329 suggesting that RNase H2-deficient cells should be killed by the ATRi/PARPi

330 combination within the $48-72$ hours needed to induce RC. Indeed, a 72 -hour treatment

331 with combined RP-3500 and niraparib followed by removal of the compounds was as

332 lethal to 5637 RNASEH2B-KO cells as a continuous, 7-day exposure or continuous

333 niraparib combined with 72 hours of RP-3500 (Figure 5G,H). Consistent with these

334 observations, we detected apoptosis marked by caspase-3 cleavage in a subset of

335 RNASEH2B-KO cells treated with RP-3500 and niraparib for 72 hours (Supplementary

336 Figure 7D,E). Taken together, our results show that a 72-hour treatment with

337 ATRi/PARPi is sufficient to induce RC and cell death in cells lacking RNase H2. Based

338 on these results, we hypothesized that a weekly treatment schedule of intermittent 3 
339 days on/4 days off may be efficacious against RNase H2-deficient tumors in vivo and

340 may circumvent the toxicity caused by continuous dosing of ATRi/PARPi (Fang et al.,

$3412019)$ by limiting the exposure of normal proliferating tissues to the combination.

343 In vivo activity of ATRi/PARPi combinations

344 To test whether tumors with RNase H2 loss are sensitive to a low-dose RP-3500/PARPi

345 combination administered at an intermittent schedule in vivo, we developed the isogenic

346 DLD1 WT and RNASEH2B-KO cells as subcutaneous xenografts in

347 immunocompromised mice. We chose PARPi talazoparib in this study due to its high

348 potency and PARP trapping activity (Murai et al., 2014; Shen et al., 2013) and because

349 it has shown in vivo efficacy in an RNase H2-deficient model (Zimmermann et al.,

350 2018). However, the relatively long in vivo half-life of talazoparib (de Bono et al., 2017;

351 Stewart et al., 2014) makes its intermittent dosing impractical. Since we saw no

352 difference between 72-hour dosing of both agents and 72-hour dosing of RP-3500

353 combined with continuous PARPi in vitro (Figure 5G,H), we evaluated a schedule of

354 continuous talazoparib and RP-3500 dosed intermittently (Figure 6A; note that the

355 longer treatment of RNASEH2B-KO tumors was to accommodate the slightly slower

356 growth of this model compared to WT). Single agent talazoparib and RP-3500 were

357 dosed at their maximal tolerated doses (MTD), as well as 1/2 MTD of talazoparib or 1/3

358 MTD of RP-3500 and a combination of the lower doses. The growth of RNASEH2B-KO

359 tumors was significantly inhibited by single-agent talazoparib at the MTD, and by RP-

3603500 at both MTD and the 1/3 lower dose ( $56 \%, \sim 69 \%$ and $~ 55 \%$ tumor growth 
361 inhibition (TGI) respectively, Figure 6A) as expected (Wang et al., 2019; Zimmermann

362 et al., 2018). Importantly, the combination of 1/2 MTD talazoparib and 1/3 MTD RP-

3633500 led to a marked TGI of $\sim 83 \%$, which was significantly different from talazoparib at

364 its MTD (Figure 6A). At the same time, all agents showed no or only mild activity in

365 RNase H2-proficient tumors (Figure 6A). All treatments were well tolerated with $<10 \%$

366 mean body weight loss (Supplementary Figure 8A).

Next, we tested the efficacy of ATRi/PARPi in a clinically relevant model with loss

of RNase H2. Using immunohistochemistry (IHC) with a pan-RNase $\mathrm{H} 2$ antibody (Reijns

369 et al., 2012) we identified a patient-derived triple-negative breast cancer tumor

370 xenograft (PDX) without detectable RNase H2 expression (Supplementary Figure 8B)

371 and tested the dosing regimens as described above. Single agent talazoparib did not

372 impact growth, but we did observe a dose-dependent tumor growth inhibition with RP-

3733500 (Figure 6B). Strikingly, the combination of 1/2 MTD talazoparib and 1/3 MTD RP-

3743500 led to tumor regression with three of nine mice being tumor free at day 40 as

375 compared to an absence of tumor-free mice in other treatment groups (Figure 6B). All

376 treatments were well tolerated with $<5 \%$ body weight loss (Supplementary Figure 8 C).

377 Taken together, these data show that RNase H2-deficient tumors are sensitive to

378 ATRi/PARPi combinations in vivo and that this sensitivity allows the use of lower doses

379 and intermittent dosing schedules that are well tolerated.

Finally, we tested the in vivo activity of a RP-3500/PARPi combination in an

$381 \mathrm{ALT}+$ xenograft tumor model generated from the U2OS cell line. In this case we used

382 the PARPi niraparib, which has a shorter half-life than talazoparib in mice (Jones et al.,

383 2015), and allowed us to directly test a 3 days on/4 days off intermittent dosing 
384 schedule. Whereas RP-3500 and niraparib at their MTDs showed only modest efficacy

$385(\sim 76 \%$ and $\sim 56 \%$ TGI, respectively, Figure $6 \mathrm{C})$, all combination dosing schedules

386 showed complete tumor regression, with sustained responses for $\geq 20$ days post

387 treatment cessation. Seven of 8 mice were tumor free at day 50 in the $15 \mathrm{mg} / \mathrm{kg}(1 / 2$

388 MTD) RP-3500 + 50 mg/kg ( 2/3 MTD) niraparib group, although tumors eventually re-

389 grew (Figure 6C). No treatments decreased body weight significantly (Supplementary

390 Figure $8 \mathrm{D}$ ). In summary, these data show that full regression of $A L T+$ tumors can be

391 achieved with ATRi/PARPi combinations in a mouse model. 


\section{DISCUSSION}

394 PARPi/ATRi combinations hold great promise for cancer therapy. These two classes of

395 agents synergistically enhance each other's activity against tumor cells and may

396 potentially avoid, delay or overcome therapy resistance (Kim et al., 2020, 2017; Lloyd et

397 al., 2020; Murai et al., 2016; Schoonen et al., 2019; Yap et al., 2016; Yazinski et al.,

398 2017). However, tolerability of the combination at monotherapy MTDs is limited by

399 hematological toxicities (Yap et al., 2016), requiring dose reductions that may impact

400 efficacy. Consequently, there is a clear need to consider alternative strategies to

401 harness the potential of this combination. We contend that these include the

402 development of optimized dosing schedules and/or the identification of sensitivity

403 biomarkers that allow the use of lower doses to improve tolerability, without

404 compromising efficacy.

Here we performed a genome-wide chemogenomic CRISPR/Cas9-based screen

406 to map the cellular response to the novel ATRi RP-3500 as a single agent or in

407 combination with a PARPi. As our screens complement previous datasets (Bajrami et

408 al., 2014; Hustedt et al., 2019; Olivieri et al., 2020; Wang et al., 2019; Zimmermann et

409 al., 2018) we provide it as a public resource, as we believe the data can yield novel

410 biological insights and inform the clinical development of ATRi and ATRi/PARPi

411 combinations. Based on data gleaned here, combined with a proprietary analysis of

412 cancer genomic datasets, we selected 17 ATRi-sensitizing gene mutations (validated

413 here and previously (Hustedt et al., 2019; Wang et al., 2019)) as a basis for patient

414 selection in our first-in-human RP-3500 clinical study (NCT04497116). 
In our chemogenomic screen we identified and subsequently validated multiple

416 cancer-relevant genetic alterations that profoundly sensitized cells to ATRi/PARPi

417 combinations and may constitute potential clinical biomarkers. Other preclinical studies

418 have identified additional biomarkers for ATRi/PARPi combinations, such as deleterious

419 alterations in BRCA1/2 (Kim et al., 2020, 2017; Schoonen et al., 2019) or ATM (Lloyd et

420 al., 2020), Cyclin E overexpression in breast and ovarian cancer (Kim et al., 2020),

421 PAX3-FOXO1 fusion in alveolar rhabdomyosarcoma (García et al., 2020), or combined

422 TP53/RB1 loss in metastatic prostate cancer (Nyquist et al., 2020).

The first cancer-relevant genetic alteration identified here was loss of RNase $\mathrm{H} 2$,

424 which expands on previously reported findings of single agent PARPi and ATRi

425 sensitivity (Wang et al., 2019; Zimmermann et al., 2018). We demonstrate that RP-

426 3500/PARPi combinations synergize and induce cell death in RNase H2-deficient cells

427 at concentrations that are almost benign in cells with functional RNase $\mathrm{H} 2$. Furthermore,

428 low-dose RP-3500/PARPi combinations show robust in vivo efficacy in RNase H2-

429 deficient xenograft models. Loss of RNase $\mathrm{H} 2$ is found in a subset of chronic

430 lymphocytic leukemias (CLL) (Zimmermann et al., 2018); therefore, an important

431 avenue for further study is to test the ATRi/PARPi combinations in preclinical CLL

432 models (Herman and Wiestner, 2016; Kwok et al., 2016; Zimmermann et al., 2018).

433 Loss of RNASEH2B was also reported in metastatic prostate cancer, although

434 homozygous deletions of RNASEH2B in this context are likely rare (Wang et al., 2019;

435 Zimmermann et al., 2018). It will be crucial to determine if $R N A S E H 2 B$ loss can be

436 detected in additional tumor types and whether these tumors can respond to

437 ATRi/PARPi combinations. We have identified a TNBC PDX model with no RNase H2 
438 expression by IHC, suggesting that RNase $\mathrm{H} 2$ loss can be detected outside the context

439 of CLL. Of note, while this manuscript was under preparation, Miao et al. reported, in a

440 pre-print, robust efficacy with an ATRi/PARPi combination in RNase H2-deficient

441 prostate cancer models, which was well in agreement with the results of our study (Miao

442 et al., 2021).

Using RNase H2-deficient cells as an example, we showed that the ATRi/PARPi

444 combination can lead to RPA exhaustion and RC. In BRCA1/2- or ATM-deficient cells,

445 the potentiation of the PARPi effects by ATRi is attributed primarily to unscheduled entry

446 into mitosis due to abrogation of the G2/M phase checkpoint (Kim et al., 2017;

447 Schoonen et al., 2019). Therefore, RNase H2 deficiency is to our knowledge, the first

448 context in which $\mathrm{RC}$ contributes to the synergy between ATRi/PARPi. This raises the

449 possibility that PARPi/ATRi combinations can kill cells by multiple (but not necessarily

450 mutually exclusive) mechanisms dependent on the respective genetic context.

451 Importantly, we used our mechanistic findings to design an optimized, 3 days on/4 days

452 off weekly intermittent schedule for in vivo dosing of RP-3500/PARPi combinations and

453 demonstrated that this schedule is effective and well tolerated in animal models. We are

454 currently evaluating this and related schedules in Phase I clinical trials (NCT04497116,

455 NCT04972110).

$456 \quad$ Next we showed ATRi/PARPi sensitivity upon inactivation of RAD51 paralogs

$457 R A D 51 B, C$ and $D$. Interestingly, cells lacking $R A D 51 B$ have been shown to be less

458 sensitive to PARPi than cells lacking $R A D 51 C$ and $D$, which has been attributed to a

459 differential requirement of these factors for HR (Garcin et al., 2019). In contrast, we

460 observed that ATRi sensitivity after loss of all three paralogs was comparable, as was 
461 the sensitivity to ATRi/PARPi, which cannot be explained by a simple difference in

462 requirement for each paralog for HR. RAD51 paralogs also play important roles in

463 replication fork maintenance and DNA damage tolerance (Berti et al., 2020; Rein et al.,

464 2021; Saxena et al., 2019; Somyajit et al., 2020), and it is therefore tempting to

465 speculate that the difference in sensitivity to ATRi and PARPi between individual

466 RAD51 paralogs may be governed by these non-HR functions, although further

467 experimental evidence is needed to support this hypothesis. In a more general sense,

468 our observations highlight the need to establish bona fide clinical biomarkers for ATRi. It

469 is apparent from our screen that, despite substantial (and expected) overlap between

470 the ATRi and PARPi treatment arms, some genes and pathways preferentially respond

471 to one treatment over another, and it will be important to establish whether clinical

472 biomarkers for PARPi will be universally applicable to ATRi and vice versa.

As a third cancer alteration that sensitizes cells to ATRi/PARPi combinations, we

474 validated ALT. A functional link between ALT and ATRi sensitivity was reported

475 previously, although it remained somewhat controversial (Deeg et al., 2016; Flynn et al.,

476 2015). We identified several ALT-associated factors in our ATRi/PARPi screen and

477 showed that ATRi/PARPi treatment elevates markers of ALT activity, suggesting that

478 the effects of ATRi/PARPi in ALT+ cell lines are due to a direct impact on ALT. In future

479 research it will be important to establish which ATR and PARP targets impact ALT

480 activity; however, ATR-regulated replication fork maintenance factors such as

481 SMARCAL1 or FANCM are obvious candidates (Bansbach et al., 2009; Singh et al.,

482 2013). Interestingly, we showed a strong and durable response of an ALT+ mouse

483 xenograft to the RP-3500/PARPi combination, despite this model showing only 
484 moderate responses to each single agent, which highlights the synergistic potential of

485 ATRi/PARPi combinations. It will be important to determine whether other genetic

486 lesions in cancer can create such a profound vulnerability to ATRi/PARPi (and other

487 DDR agent combinations), despite leading to only modest single-agent activity.

In conclusion, we demonstrate that using CRISPR chemogenomic screening

489 combined with mechanistic insights can guide the rational use of drug combinations in

490 preclinical models to minimize toxicity while preserving the efficacy of the combination,

491 and believe that the findings reported here will inform investigation of ATRi/PARPi

492 combination regimens in the clinic.

\section{ACKNOWLEDGEMENTS}

495 We thank Daniel Durocher and members of the Repare Therapeutics team for critical

496 reading of the manuscript and Daniel Durocher and Andrew Jackson for reagents.

\section{AUTHOR CONTRUBUTIONS}

498 M. Zimmermann conceived the study with input from AR and M. Zinda. M. Zimmermann

499 also performed most cell biology experiments, analyzed data and wrote the paper with

500 input from other authors. CB and JD performed the CRISPR screen under supervision

501 from JTFY. BK and AS performed cell biology assays. SF and LL conducted in vivo

502 mouse studies with supervision from AR. AV analyzed CRISPR screening and PDX

503 genomic data. VR directed IHC experiments. M. Zinda supervised all activities and

504 helped write the manuscript. 


\section{DECLARATION OF INTERESTS}

506 All authors except AS are current or former employees of Repare Therapeutics and

507 receive salary and equity compensation. AS received salary from Repare Therapeutics

508 as part of this work. 
511 Figure 1. CRISPR screen for sensitizers to RP-3500 + PARPi.

512 A. Screen design. Cas9-expressing RPE1 cells were transduced with the TKOv3 library

513 at day 0 and treated with DMSO or $L_{20}$ doses of each RP-3500, niraparib or

514 combination from days 6 to 18. sgRNA representation at the initial and final timepoints

515 was quantified by NGS.

516 B. A Venn diagram showing the number of high-confidence (Jenks class $3+4$ ) hits in the

517 respective treatment arms and GO pathway enrichment in each dataset. Genes scoring 518 in all three arms are highlighted. See also Supplementary Figure 1.

520 Figure 2. RNase H2-deficient cells are sensitive to ATRi/PARPi.

521 A,B. Loss of RNase H2 subunits in RNASEH2B-KO cells. Representative (of $\geq 2$

522 independent biological replicates) immunoblots of 5637 (A) or DLD1 (B) WT and

523 RNASEH2B-KO cells. Whole cell lysates were processed for immunoblotting with

524 indicated antibodies. $\alpha$ ACTININ, loading control.

525 C-E. Viability of 5637 or DLD1 cells of indicated genotypes treated with either RP-3500

526 (ATRi), PARPi talazoparib or niraparib, or a RP-3500+PARPi combination; values are

527 normalized to untreated controls. Doses of compounds are shown below panels. Circles

528 are values from three independent biological replicates; Bars indicate mean $\pm S D$. $P$

529 values calculated with a two-tailed unpaired Student's t-test. 
530 F-H. Zero interaction potency (ZIP) synergy scores at various dose combinations of RP-

5313500 and PARPi in cell lines of indicated genotypes. Score $\geq 10$ (red) represents

532 synergy, $\leq-10$ (green) antagonism. Dashed lines mark doses that show maximal

533 synergy. Values obtained by analyzing mean data from three independent biological

534 replicates with SynergyFinder. See also Supplementary Figure 2 and 3.

536 Figure 3. Differential requirement of RAD51 paralogs in response to PARPi and 537 ATRi.

538 A-D. Single agent ATRi (RP-3500, A,B) and PARPi (olaparib, C,D) sensitivity in cells

539 deficient for RAD51 paralogs. A,C. Dose-response curves of Cas9-expressing RPE1

540 cells transduced with indicated sgRNAs. Data points are mean of $\geq 3$ independent

541 biological replicates $\pm S D$, normalized to untreated controls. Solid lines show a non-

542 linear least square-fit to a four-parameter dose-response model. B,D. RP-3500 and

543 olaparib $\mathrm{IC}_{50}$ values in cells transduced with the indicated sgRNAs. Values obtained

544 from non-linear least square fitting of data from three independent biological replicates

545 as shown in $\mathbf{A}$ and $\mathbf{C}$. Error bars represent a $95 \%$ confidence interval $(\mathrm{Cl})$.

546 E. Combination sensitivity. Viability of sgRNA-transduced RPE1 cells after indicated

547 treatments; values are normalized to untreated controls. Doses of compounds are

548 shown below panels. Circles are values from three independent biological replicates;

549 Bars indicate mean \pm SD. 
550 F. ZIP synergy scores at various dose combinations of RP-3500 and olaparib in cells

551 transduced with indicated sgRNAs. Score $\geq 10$ (red) represents synergy, $\leq-10$ (green)

552 antagonism. Dashed lines mark doses showing maximal synergy. Values obtained by

553 analyzing mean data from three independent biological replicates with SynergyFinder.

554 See also Supplementary Figure 4.

556 Figure 4. ALT+ cells are sensitive to RP-3500+PARPi.

557 A. Representative ssTeloC micrographs showing ALT activity status in the indicated cell

558 lines. ssTeloC foci (green), Alexa Fluor 488; DAPI (blue), nuclear counterstain.

559 B. Quantification of mean ssTeloC focus fluorescence intensity in the indicated cell

560 lines. Circles represent mean focus intensity per replicate ( $N=3$ biological replicates,

561 except SAOS2, $\mathrm{N}=2$ ). Bars show mean $\pm \mathrm{SD}$.

562 C. Left: Quantification of viability (CellTiter Glo) of the indicated cell lines after treatment

563 with $5 \mathrm{nM} \mathrm{RP-3500} \mathrm{(ATRi),} 5 \mathrm{nM}$ talazoparib (PARPi) or the combination. Data

564 normalized to untreated cells. TEL $+=$ telomerase-positive, $A L T+=A L T-$ positive. Circles

565 represent data from individual biological replicates $(N \geq 3)$, bars indicate mean $\pm S D$.

566 Right: Summary view of mean (of three or more biological replicates) combination-

567 treated cell viability in the indicated cohorts. Each data point represents a cell line.

568 D. ATRi/PARPi combination elevates ALT activity in a sensitive cell line. Quantification

569 of mean ssTeloC focus fluorescence intensity in a ATRi/PARPi sensitive (U2OS, left)

570 and resistant (SAOS2, right) ALT+ cell line. Circles represent mean focus intensity per 
571 replicate ( $\mathrm{N}=3$ biological replicates). Bars show mean $\pm \mathrm{SD}$. $P$ value calculated with a

572 two-tailed unpaired Student's t-test. See also Supplementary Figure 5.

574 Figure 5. ATRi/PARPi cause irreparable DNA damage and RPA exhaustion in 575 RNase H2-null cells.

576 A. Timeline of experiments shown in B-C. 5637 WT or RNASEH2B-KO cells were

577 treated as indicated for 72 hours followed by immediate processing for

578 immunofluorescence or a washout and a recovery period for indicated time points.

579 B,C. Quantification of cells positive (+) for pan-nuclear $\gamma-\mathrm{H} 2 \mathrm{AX}(\mathbf{B})$ or EdU (C) after 580 indicated treatments and at time points as in $\mathbf{A}$.

581 D. Timeline of experiments shown in E,F.

582 E. Quantification of immunofluorescence signals of chromatin-bound RPA32 and $\gamma$ -

$583 \mathrm{H} 2 \mathrm{AX}$ in $5637 \mathrm{WT}$ and RNASEH2B-KO cells treated as shown in D. Each point

584 represents a single nucleus, solid lines show gates for $\gamma-\mathrm{H} 2 \mathrm{AX}+$ and RPA32+ cells.

585 Replication catastrophe $(\mathrm{RC})+$ cells $(\mathrm{RPA} 32+/ \gamma-\mathrm{H} 2 \mathrm{AX}+)$ are outlined with red

586 rectangles. Representative plots of three independent biological replicates.

587 F. Percentages of RC+ cells.

588 G,H. Short-term ATRi/PARPi treatment is sufficient to kill RNase H2-null cells. G.

589 Timeline of the experiment shown in H. $5637 \mathrm{WT}$ or RNASEH2B-KO cells were treated 590 with RP-3500, niraparib or the combination at the indicated schedules in a 7-day 
591 CellTiter Glo viability assay. H. Quantification of cell viability in experiments outlined in

592 G.

593 Data in $\mathbf{B}, \mathbf{C}, \mathbf{F}, \mathbf{H}$ are represented as follows: Circles, values from three independent

594 biological replicates; Bars, mean $\pm S D$. $P$ values calculated with a two-tailed unpaired

595 Student's t-test.

597 Figure 6. Low dose talazoparib combined with RP-3500 treatment shows efficacy 598 in xenograft models of sensitive genetic backgrounds.

599 A. Tumor growth of DLD1 WT and DLD1 RNASEH2B-KO cell lines in NOD-SCID mice 600 treated with talazoparib, RP-3500 or both. Talazoparib was administered QDx7 weekly 601 and RP-3500 QDx3 weekly starting on Day 1 of each week. Results are expressed as 602 mean \pm SEM, $\mathrm{N}=8$ mice/group and treatment continued for 14 and 16 days for DLD1 603 WT and DLD1 RNASEH2B-KO, respectively.

604 B. Tumor growth of a triple negative breast cancer patient-derived PDX in Balb/C nude 605 mice. Talazoparib was administered QDx7 weekly and RP-3500 QDx3 weekly starting 606 on day 2 of every week for 4 weeks.

607 C. Tumor growth of U2OS cells in NCG mice treated with niraparib and/or RP-3500 608 QDx3; niraparib was also given at its MTD of $70 \mathrm{mg} / \mathrm{kg}$ QDx5 for comparison. Results 609 are expressed as mean $\pm \mathrm{SEM}, \mathrm{N}=8$ mice/group. 
$610 P$ values were determined from tumor volumes by one-way analysis of variance

611 (ANOVA) using the Fisher's least significant difference (LSD) post-test. See also

612 Supplementary Figure 8.

613 


\section{MATERIALS AND METHODS}

\section{Cell culture and generation of CRISPR KO cell lines}

616 5637, DLD1, RPE1-hTERT, MCF10A, HELA, HOS, HS683, HT1080, SJSA1, HS729T,

617 SAOS2 and U2OS cell lines were purchased from ATCC. CAL72 cells were obtained

618 from DSMZ. COL-hTERT (Immortalized Human Colon Cells) were purchased from

619 ABM. TM31 were obtained from RIKEN BioResource Research Center. 5637, DLD1

620 and SJSA1 cells were cultured in RPMI-1640 medium (Corning 10-040-CV)

621 supplemented with $10 \%$ fetal bovine serum (FBS; VWR 97068-085), 100U/mL penicillin

622 and 100 $\mathrm{g} / \mathrm{mL}$ streptomycin (Pen/Strep; Corning 30-001-CI). RPE1, TM31, HELA,

623 HT1080, HS729T cells were cultured in Dulbecco's Modified Eagle Medium (DMEM;

624 Corning 10-014-CV) supplemented with 10\% FBS and Pen/Strep. SAOS2 and U2OS

625 cells were cultured in McCoy's 5A medium (Cytiva SH30200.FS) supplemented with

$62610 \%$ FBS and Pen/Strep. All cell lines were maintained at $37^{\circ} \mathrm{C}$ and $5 \% \mathrm{CO}_{2}$.

628 GenScript USA, Inc., using a 5' AAGAGAACTTACCTGAACAG 3' sgRNA target

629 sequence. Briefly, Cas9/sgRNA nucleoprotein complexes were transfected into cells

630 and single clones were isolated by limiting dilution. Clones were screened using

631 polymerase chain reaction (PCR, forward primer 5' ACCCCTGCTTCTCATCATTCC 3';

632 reverse primer 5' TTGCCCGTATTTCTGATGGCT 3') and TIDE analysis (Brinkman et

633 al., 2014) as well as immunoblotting. RPE1-hTERT Cas9 TP53-KO and RPE1-hTERT

634 Cas9 TP53-KO RNASEH2B-KO cells were described previously (Zimmermann et al., 635 2018). 


\section{Chemical compounds}

637 Stock solutions of RP-3500 (synthesized by OmegaChem), olaparib, talazoparib and

638 niraparib (all MedChem Express) were made up from powder in DMSO and kept at $639-20^{\circ} \mathrm{C}$ for long-term storage.

641 CRISPR/Cas9-enabled chemogenomic screening

642 CRISPR screens were performed according to a published procedure (Olivieri and

643 Durocher, 2021). On day -3, Cas9-expressing RPE1-hTERT TP53-KO cells were

644 infected with the TKOv3 lentiviral library (Hart et al., 2017) at a low multiplicity of

645 infection (MOI; 0.6) and transductants were selected with puromycin from day -2 to

646 day 0 . On day 0 transduced cell pools were split into two technical replicates and

647 cultured until day 6 . On day 6 , each replicate was split into four treatment arms - DMSO

648 control, RP-3500, niraparib and a RP-3500+niraparib combination. The following

649 concentrations were used, which resulted in a $\sim 20 \%$ loss of cell viability (an $L D_{20}$ dose)

650 at the screen: $15 \mathrm{nM}$ RP-3500 single agent, $1 \mu \mathrm{M}$ niraparib single agent and a

651 combination of $8 \mathrm{nM}$ RP-3500 with 150nM niraparib. Cells were cultured in the presence

652 of compounds until day 18 . Genomic DNA was isolated from day 0 and day 18 samples,

653 sgRNA genomic integrants were amplified by PCR and sgRNA representation in each

654 sample was quantified by next generation sequencing. Data were analyzed using the

655 CCA algorithm (Adam et al., 2021). 


\section{Lentiviral sgRNA transduction}

658 Individual TKOv3 sgRNAs were cloned into a lentiGuide-NLS-GFP vector (Noordermeer

659 et al., 2018). Lentiviral stocks were prepared by seeding $18 \times 10^{6} 293$ T LentiX cells on a

$66015 \mathrm{~cm}$ dish in $20 \mathrm{~mL}$ media and transfected 24 hours later by adding $2.4 \mathrm{~mL}$ of

661 transfection mix, which contained OptiMEM media, $18 \mu \mathrm{g}$ of sgRNA plasmid, lentiviral

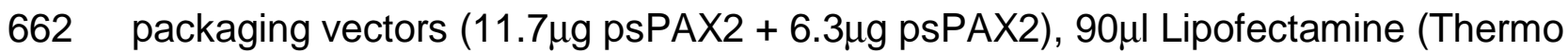

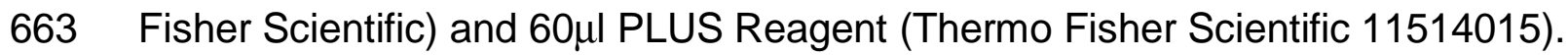

664 Medium was exchanged 16-20 hours after transfection and supplemented with 20mM

665 HEPES. Virus-containing supernatant was collected $\sim 48$ hours post transfection,

666 cleared by centrifugation at 1000 RPM for 5 minutes and stored at $-80^{\circ} \mathrm{C}$.

$6671 \times 10^{5}$ RPE1-hTERT Cas9 TP53-KO cells were transduced with the virus at MOI $\sim 1$.

668 Virus was removed 24 hours post infection and sgRNA-expressing cells were selected

669 with puromycin-containing media for 48 hours. Transduced cells were seeded for

670 experiments 7 days post infection.

671

672 CellTiter Glo cell viability assays

673 On day 0 , cells were seeded on 96-well plates (Corning, 3903). The following cell

674 numbers and assay lengths were used: 5637 WT: 300 cells/well, 7 days; 5637

675 RNASEH2B-KO: 800 cells/well, 7 days; RPE1-hTERT Cas9 TP53-KO: 200 cells/well, 6

676 days; RPE1-hTERT Cas9 TP53-KO RNASEH2B-KO: 200 cells/well, 6 days; DLD1 WT:

677300 cells/well, 8 days; DLD1 RNASEH2B-KO, 300 cells/well, 9 days.; RPE1-hTERT 
678 Cas9 TP53-KO infected with lentiviral sgRNAs: 600-800 cells/well, 7 days; HELA: 1000

679 cells/well, 7 days; HOS: 1000 cells/well, 7 days; HS683: 800 cells/well, 15 days;

680 HT1080: 800 cells/well, 7 days; SJSA1: 800 cells/well, 7 days; CAL72: 1000 cells/well, 9

681 days; HS729T: 1000 cells/well, 14 days; SAOS2: 800 cells/well, 14 days; TM31: 1000

682 cells/well, 12 days; U2OS: 800 cells/well, 7days. Compounds were added from DMSO

683 stock solutions on day 1 using a Tecan D300E dispenser (Tecan). Cells were either

684 grown in continuous presence of compounds (medium and compounds were refreshed

685 every 3-4 days) or at various schedules indicated in the respective figures (see results

686 section). Cell viability was measured using the CellTiter Glo assay kit (Promega)

687 according to manufacturer's instructions. Luminescence was read out either on Envision

6882105 (Perkin-Elmer) or FlexStation 3 (Molecular Devices) plate readers. Relative cell

689 viability was calculated by subtracting blank luminescence from each measured value,

690 averaging technical replicates and normalizing to untreated cells. Synergy between RP-

6913500 and PARPi was analyzed with the on-line SynergyFinder tool (lanevski et al.,

692 2020) using the ZIP (Yadav et al., 2015) model (https://synergyfinder.fimm.fi).

693

694 Immunoblotting

695 Whole cell lysates were prepared by resuspending cell pellets in 2 x Sample Buffer

696 (Novex Tris-Glycine SDS Sample Buffer, ThermoFisher \#LC2676, supplemented with

$697200 \mathrm{mM}$ DTT) at a concentration of $1 \times 10^{7}$ cells $/ \mathrm{mL}$. Lysates were boiled at $95^{\circ} \mathrm{C}$ for 5

698 minutes and 15-30 $\mu$ l was separated on Tris-Glycine SDS-PAGE gels (ThermoFisher)

699 followed by Western blotting onto nitrocellulose membranes (ThermoFisher, PB7220) in 
7001 1x Novex Tris-Glycine Transfer Buffer (ThermoFisher, LC3675) containing 20\%

701 methanol and $0.04 \%$ SDS. Membranes were blocked in 5\% milk / Tris-buffered saline /

$7020.1 \%$ Tween 20 (5\% milk / TBST) and incubated with primary antibodies diluted in 5\%

703 milk / TBST either overnight at $4^{\circ} \mathrm{C}$ or for 2 hours at room temperature (RT).

704 Membranes were washed for $3 \times 5$ minutes with TBST and incubated for 1 hour at RT

705 with horseradish peroxidase-conjugated secondary antibodies (goat anti-rabbit IgG,

706 Jackson ImmunoResearch 111-035-144 or sheep anti-mouse IgG, GE Healthcare

707 NA931V) diluted 1:5000 in 5\% milk / TBST. Membranes were washed as above,

708 developed with a SuperSignal West Femto chemiluminescence reagent (ThermoFisher,

709 PI34095) for 2 minutes and scanned on a ChemiDoc imager (Bio-Rad).

\section{High-Content Fluorescence Microscopy}

712 Cells were grown on CellCarrier Ultra 96-well plates (Perkin Elmer, 6055302) and

713 subjected to various treatments as indicated in the respective figures. If applicable,

$71410 \mu \mathrm{M}$ EdU was added 30 minutes before fixation to monitor DNA replication. Medium

715 was subsequently removed and cells were rinsed with phosphate buffered saline (PBS).

716 In experiments analyzing the levels of chromatin-bound RPA32 and $\gamma-\mathrm{H} 2 \mathrm{AX}$ (Figure

717 5D-F, Supplementary Figure 7), plates were placed on ice and soluble nuclear

718 proteins were extracted with ice-cold cytoskeleton (CSK) buffer (10mM PIPES pH 7.0,

$719300 \mathrm{mM}$ sucrose, $100 \mathrm{mM} \mathrm{NaCl}, 3 \mathrm{mM} \mathrm{MgCl}_{2}$ ) for 15 minutes followed by a rinse with

720 PBS. In all other experiments this CSK pre-extraction step was omitted. Cells were then

721 fixed with 4\% paraformaldehyde (PFA) in PBS for 10 minutes at RT and permeabilized 
722 with $0.3 \%$ Triton X-100/PBS for 30 minutes at RT (permeabilization was omitted if CSK

723 extraction was performed before fixation). Cells were subsequently blocked with

724 PBS/bovine serum albumin/gelatin (PBG: $0.2 \%$ cold water fish gelatin, $0.5 \%$ bovine

725 serum albumin [BSA] in PBS) for 30 minutes at RT and incubated with primary

726 antibodies diluted in PBG for 2 hours at RT. Afterwards cells were rinsed twice with PBS

727 and incubated with fluorescently labeled secondary antibodies (Alexa Fluor 488 or 555-

728 conjugated goat anti-rabbit IgG or goat anti-mouse IgG, Invitrogen) in PBG for 1 hour at

729 RT. $0.5 \mu \mathrm{g} / \mathrm{mL}$ DAPI was included with secondary antibodies as a nuclear counterstain.

730 Cells were finally rinsed $3 x$ with PBS and either immediately imaged or processed for

731 click chemistry to visualize DNA-incorporated EdU. For EdU staining, cells were fixed

732 again with 4\% PFA/PBS for 5 minutes at RT and rinsed with PBS. Click chemistry was

733 then performed using a Click-iT EdU Cell Proliferation Kit for Imaging, Alexa 647

734 (ThermoFisher, C10340), according to the manufacturer's instructions. Plates were

735 imaged on an Operetta automated microscope (Perkin Elmer) in confocal mode and

736 image analysis was performed in the Harmony software (Perkin Elmer). Examples of

737 gating cells positive for the respective markers is shown in Figure 5E and

738 Supplementary Figures 6,7.

740 ssTeloC assay

741 The ssTeloC assay was performed as described previously (Loe et al., 2020). Cells

742 were seeded on CellCarrier Ultra 96-well plates (Perkin Elmer, 6055302) and, if desired,

743 treated with RP-3500, talazoparib, or their combination for 24 hours. Medium was 
744 removed, cells were rinsed with PBS and fixed with 2\% PFA/PBS for 5 minutes at RT.

745 The plates were then washed with PBS and incubated with RNase A blocking solution

$746(500 \mu \mathrm{g} / \mathrm{mL}$ RNase A, $1 \mathrm{mg} / \mathrm{mL}$ BSA, $3 \%$ normal goat serum, $0.1 \%$ Triton X-100, $1 \mathrm{mM}$

747 EDTA in PBS) for 1 hour at $37^{\circ} \mathrm{C}$. Samples were subsequently dehydrated with a $70 \%$,

$74895 \%$ and $100 \%$ ethanol series (5 minutes, RT, each) and allowed to air-dry. Native

749 fluorescence in situ hybridization (FISH) was performed by incubating cells with a

750 hybridization mix ( $70 \%$ formamide, $1 \mathrm{mg} / \mathrm{mL}$ Roche FISH blocking reagent

751 11096176001, 10mM Tris/HCl pH 7.0) containing an Alexa Fluor 488-labeled TelG PNA

752 probe (PNA BIO F1008) at a 1:500 concentration for 2 hours at RT. Plates were

753 subsequently washed $2 x$ with $70 \%$ formamide/PBS for 15 minutes each and $3 \times 5$

754 minutes with PBS. $0.5 \mu \mathrm{g} / \mathrm{mL}$ DAPI was included in the second PBS wash. Plates were

755 imaged on an Operetta automated microscope (Perkin Elmer) in confocal mode and

756 image analysis was performed in the Harmony software (Perkin Elmer).

758 Antibodies

759 The following primary antibodies were used for immunoblotting (IB) and

760 immunofluorescence (IF): Mouse anti-RNASEH2A G-10 (SCBT sc-515475, IB 1:50),

761 rabbit anti-RNASEH2B (Novus NBP2-58962, IB 1:200), rabbit anti-RNASEH2C

762 (Proteintech 16518-1-AP, IB 1:1000), rabbit anti-pCHK1(S345) 133D3 (CST 2348, IB

763 1:1000), mouse anti-CHK1 2G1D5 (CST 2360, IB 1:200), mouse anti- $\alpha$ ACTININ

764 AT6/172 (Millipore Sigma 05-384, IB 1:5000), mouse anti- $\gamma$-H2AX JBW301 (Millipore

765 Sigma 05-636, IF 1:2000), rabbit anti- $\gamma-\mathrm{H} 2 \mathrm{AX}$ (CST 2577, IF 1:500), mouse anti-RPA32 
766 (Abcam ab2175, IF 1:1000), rabbit anti-cleaved caspase-3 (CST 9664, IF 1:1000),

767 mouse anti-pH3(S10) (ThermoFisher, MA515220, IF 1:1000).

\section{Immunohistochemistry}

770 Formalin-fixed, paraffin-embedded PDX tumor samples were obtained from Crown Bio.

771 RNase $\mathrm{H} 2$ protein expression was assessed by IHC using a sheep polyclonal anti-

772 RNase H2 antibody (Reijns et al., 2012) (a gift from Andrew Jackson) at 1:300 and

773 rabbit anti sheep IgG $(H+L)$ secondary link antibody (Invitrogen \#31240 1:4000)

774 following Leica BondRx standard IHC protocols. PDX models were considered RNase

775 H2-negative if they showed a complete absence of RNase H2 staining.

\section{Mouse studies}

778 DLD1 and DLD1 RNASEH2B-KO cells were implanted at 5 and $10 \times 10^{6}$ cells per

779 mouse, respectively, in PBS into the right flank of female NOD-SCID mice (5-7 weeks

780 old; Charles River). U2OS cells were implanted at $1 \times 10^{7}$ cells in 50:50 PBS:Matrigel

781 (Corning, \# CB35248). When tumors had reached the target size of $100-150 \mathrm{~mm}^{3}$, mice

782 were randomized into treatment groups $(n=7-8)$. In vivo studies involving cell-derived

783 xenografts were performed in a Canadian Council on Animal Care-accredited vivarium

784 with an Institutional Animal Care Committee-approved protocol. In vivo studies using

785 PDX were conducted at Crown Biosciences Inc. (Taicang). Fresh tumor tissue from

786 mice bearing established primary human tumors were harvested and cut into small 
787 pieces (approximately $2-3 \mathrm{~mm}$ in diameter). These tumor fragments were inoculated

788 subcutaneously into the upper right dorsal flank of female BALB/c nude mice (5-7

789 weeks old) for tumor development. When the mean tumor size reached approximately

$790150(100-200) \mathrm{mm}^{3}$, mice were randomized into treatment groups $(n=6)$ according to

791 growth rate. The procedures involving the care and use of animals in this study were

792 reviewed and approved by the Institutional Animal Care and Use Committee of

793 CrownBio and were conducted in accordance with the regulations of the Association for

794 Assessment and Accreditation of Laboratory Animal Care.

795 RP-3500 and niraparib were formulated in $0.5 \%$ methylcellulose, $0.02 \%$ sodium

796 lauryl sulfate. Talazoparib was formulated in $0.5 \%$ carboxymethyl cellulose and

797 combined with RP-3500 before oral administration. Talazoparib was administered once

798 daily (QD) for a maximum of 3 weeks; RP-3500 and niraparib were administered QD for

799 three consecutive days weekly starting on Day 1 or 2 of each week. Tumor volume (TV)

800 was measured using a digital caliper and calculated using the formula $0.52 \mathrm{x}$ Length $\mathrm{x}$

801 Width ${ }^{2}$. Response to treatment was evaluated for TGI. TGI was defined as: $\%$ TGI=

802 ([TVvehicle/last - TVvehicle/day0] - [TVtreated/last - TVtreated/day0]) / (TVvehicle/last

803 - TVvehicle/day0) x100. Body weight (BW) was represented as change in BW using the

804 formula: \%BW change $=($ BWlast $/$ BWday 0$) \times 100$. BW change was calculated based

805 on individual BW changes relative to Day 0 . Statistical significance relative to vehicle

806 control was established by one-way ANOVA followed by Fisher's LSD test. 


\section{SUPPLEMENTARY FIGURE LEGENDS}

\section{Supplementary Figure 1. Related to Figure 1.}

809 A. Venn diagram showing the number of statistically significant hits $(P<0.05)$ in the 810 three CRISPR screen treatment arms.

811 B. Overlap between the RP-3500 CRISPR screen hits in this study and a published

812 'consensus' set of ATRi sensitizing genes (Hustedt et al., 2019). Common hits are listed 813 on the right.

814 C. siRNA validation of selected RP-3500 sensitizing hits. MCF10A, RPE1-hTERT TP53-

$815 \mathrm{KO}$ and HeLa cells were transfected with indicated siRNA pools and sensitivity to RP-

8163500 was determined by 5 day CellTiter Glo assays. Data are represented as a fold-

817 shift in RP-3500 IC $\mathrm{I}_{50}$ from to non-targeting siCTRL pool (circles) from $\geq 2$ independent 818 biological replicates. Bars show mean \pm SD.

819 D. CRISPR screen hits in RP-3500 single agent and RP-3500+PARPi arms as

820 determined by CCA. Each circle represents a gene, straight lines denote hit cutoffs ( $P$

$821<0.05)$. Colors indicate Jenks classes in the RP-3500+PARPi combination arm. Hits

822 discussed in this manuscript are highlighted in dark blue.

823

824

825 


\section{Supplementary Figure 2. Related to Figure 2.}

828 A. Loss of RNase H2 subunits in RPE1-hTERT TP53-KO RNASEH2B-KO cells. Whole 829 cell lysates of indicated genotypes were processed by immunoblotting with RNASEH2A, $830 \quad B$ and $C$ antibodies. $\alpha$ ACTININ was a loading control.

831 B-H. Dose-response curves of 5637, DLD1 and RPE1 cells of indicated genotypes to

832 single agent ATRi (RP-3500, B-D) or PARPi (niraparib or talazoparib, E-H). Data points 833 are mean of $\geq 3$ independent biological replicates $\pm S D$, normalized to untreated controls.

834 Solid lines show a non-linear least square-fit to a four-parameter dose-response model.

835 i-k. RP-3500, niraparib and talazoparib $\mathrm{IC}_{50}$ values (shown as bars) for various WT and 836 RNASEH2B-KO cell lines from experiments shown in B-H. Error bars indicate 95\% 837 confidence intervals from non-linear least square fitting.

839 Supplementary Figure 3. Related to Figure 2. Heat maps showing viability of 5637

840 (A,B), DLD1 (C) and RPE1 (D) WT and RNASEH2B-KO cells at indicated

841 concentrations of RP-3500 (ATRi), talazoparib (PARPi, A,C,D), niraparib (PARPi, B), or

842 their combinations. Top panels (white-to-red scale) visualize mean viability values from

843 three independent biological replicates with corresponding SDs shown in bottom panels

844 (white-to-purple scale). E. ZIP synergy scores in RPE1 TP53-KO RNASEH2B-WT and

845 TP53-KO RNASEH2B-KO cells at various dose combinations of RP-3500 and

846 talazoparib. Score $\geq 10$ (red color) represents synergy, $\leq-10$ (green) antagonism. 
847 Dashed lines mark doses showing maximal synergy. Values were obtained by analyzing

848 mean data from three independent biological replicates with SynergyFinder.

850 Supplementary Figure 4. Related to Figure 3.

851 A. CRISPR sgRNA editing efficiencies in experiments shown in Figure 3 as determined

852 by ICE (Hsiau et al., 2018). The percentages of total indel mutations as well as only out-

853 of-frame indels, which are more likely to induce a gene knockout, are shown.

854 B. Heat maps showing viability of Cas9-expressing RPE1-hTERT TP53-KO cells

855 transduced with the indicated sgRNAs treated with RP-3500 (ATRi), olaparib (PARPi) or

856 their combination. Top panels (white-to-red scale) visualize mean viability values from

857 three independent biological replicates with corresponding SDs shown in bottom panels

858 (white-to-purple scale).

859

860 Supplementary Figure 5. Related to Figure 4.

861 A. ALT-related genes highlighted on the RP-3500 and RP-3500+PARPi screen

862 diagram.

863 B. Single agent RP-3500 sensitivity of a panel of ALT-positive (ALT+, orange bars) and

864 telomerase-positive (TEL+, blue bars) cancer cell lines. Circles show RP-3500 IC 50 from

$865 \geq 3$ independent biological replicates. Bars show mean \pm SD. 


\section{Supplementary Figure 6. Related to Figure 5.}

868 A. Representative high-content microscopy images of WT and RNASEH2B-KO cells

869 stained for $\gamma-\mathrm{H} 2 \mathrm{AX}$ and EdU at the 72-hour timepoint for experiments shown in B,C and

870 Figure 5A-C. DAPI - nuclear counterstain.

871 B. Example cell cycle profiles for 5637 WT and RNASEH2B-KO cells from experiments

872 shown in Figure 5A-C. For each cell, DNA content as measured by total DAPI nuclear

873 intensity was plotted against the mean nuclear EdU intensity. Horizontal line in each

874 panel indicates the cut-off for EdU+ cells and vertical lines show the approximate peak

875 of $2 \mathrm{~N}$ and $4 \mathrm{~N}$ cell populations as determined from DNA content histograms (example

876 shown in top-most panel). Pan-nuclear $\gamma-\mathrm{H} 2 \mathrm{AX}$ cells are highlighted in red.

877 C. An example of gating strategy to quantify pan- $\gamma-\mathrm{H} 2 \mathrm{AX}+$ cells in $\mathbf{B}$ and Figure 5B.

878 DNA content was plotted against mean $\gamma-\mathrm{H} 2 \mathrm{AX}$ nuclear intensity. Horizontal lines

879 indicate the cut-off for $\gamma-\mathrm{H} 2 \mathrm{AX}+$ cells and vertical lines show the approximate peak of

$8802 \mathrm{~N}$ and $4 \mathrm{~N}$ cell populations as determined from DNA content histograms. All panels are

881 representative of three independent biological replicates.

882

883 Supplementary Figure 7. Related to Figure 5.

884 A. Timeline of experiments shown in B,C. RPE1-hTERT TP53-KO or TP53-KO

885 RNASEH2B-KO cells were subjected to indicated treatments for indicated amounts of

886 time and processed for immunostaining. 
887 B. High-content microscopy quantification of immunofluorescence signals of chromatin-

888 bound RPA32 and $\gamma-\mathrm{H} 2 \mathrm{AX}$ in cells of indicated genotypes treated as shown in A. Each

889 point represents a single nucleus, solid lines show cut-offs for $\gamma-\mathrm{H} 2 \mathrm{AX}+$ and RPA32+

890 cells. Replication catastrophe+ cells (RPA32 $+/ \gamma-\mathrm{H} 2 \mathrm{AX}+)$ are outlined with red

891 rectangles. Representative plots of three independent biological replicates.

892 C. Percentages of replication catastrophe+ cells in the indicated samples.

893 D. Representative (of three biological replicates) high-content microscopy images of

8945637 WT and RNASEH2B-KO cells stained for cleaved caspase-3 (apoptotic cells;

895 green); DAPI - nuclear counterstain.

896 E. Automated high content microscopy quantification of 5637 WT and RNASEH2B-KO

897 cells positive $(+)$ for cleaved caspase- 3 after indicated treatments and at indicated time

898 points. Data in C,E are represented as follows: Circles are values from three

899 independent biological replicates; Bars indicate mean \pm SD. $P$ value calculated with a

900 two-tailed unpaired Student's t-test.

901

902 Supplementary Figure 8. Related to Figure 6.

903 A. Tolerability of RP-3500, talazoparib and the combination in DLD1 xenograft

904 experiments in Figure 6A. Mean BW measures normalized to baseline are plotted for

905 indicated cohorts. Error bars represent \pm SEM, $N=8$ and 7 mice/group for WT and

906 RNASEH2B-KO, respectively. 
907 B. Representative IHC images of RNase H2-deficient and -proficient PDX models.

908 Deficient PDX on the top panel was used in the experiment shown in Figure 6B.

909 C. Tolerability of RP-3500, talazoparib and the combination in the PDX experiment in

910 Figure 6B. Mean BW measures normalized to baseline are plotted for indicated

911 cohorts. Error bars represent \pm SEM, $N=9$ mice/group.

912 D. Tolerability of RP-3500, niraparib and the combination in the U2OS xenograft

913 experiment in Figure 6C. Mean BW measures normalized to baseline are plotted for

914 indicated cohorts. Error bars represent \pm SEM, $N=9$ mice/group.

915

916 Supplementary Table 1 will be available at publication. 


\section{REFERENCES}

Adam, S., Rossi, S.E., Moatti, N., De Marco Zompit, M., Xue, Y., Ng, T.F., ÁlvarezQuilón, A., Desjardins, J., Bhaskaran, V., Martino, G., Setiaputra, D., Noordermeer, S.M., Ohsumi, T.K., Hustedt, N., Szilard, R.K., Chaudhary, N., Munro, M., Veloso, A., Melo, H., Yin, S.Y., Papp, R., Young, J.T.F., Zinda, M., Stucki, M., Durocher, D., 2021. The CIP2A-TOPBP1 axis safeguards chromosome stability and is a synthetic lethal target for BRCA-mutated cancer. Nat Cancer. https://doi.org/10.1038/s43018-021-00266-w

Akbari, M.R., Tonin, P., Foulkes, W.D., Ghadirian, P., Tischkowitz, M., Narod, S.A., 2010. RAD51C germline mutations in breast and ovarian cancer patients. Breast Cancer Res 12, 404. https://doi.org/10.1186/bcr2619

Bajrami, I., Frankum, J.R., Konde, A., Miller, R.E., Rehman, F.L., Brough, R., Campbell, J., Sims, D., Rafiq, R., Hooper, S., Chen, L., Kozarewa, I., Assiotis, I., Fenwick, K., Natrajan, R., Lord, C.J., Ashworth, A., 2014. Genome-wide profiling of genetic synthetic lethality identifies CDK12 as a novel determinant of PARP1/2 inhibitor sensitivity. Cancer Res 74, 287-297. https://doi.org/10.1158/0008-5472.CAN-132541

Bansbach, C.E., Bétous, R., Lovejoy, C.A., Glick, G.G., Cortez, D., 2009. The annealing helicase SMARCAL1 maintains genome integrity at stalled replication forks. Genes Dev 23, 2405-2414. https://doi.org/10.1101/gad.1839909

Berti, M., Teloni, F., Mijic, S., Ursich, S., Fuchs, J., Palumbieri, M.D., Krietsch, J., Schmid, J.A., Garcin, E.B., Gon, S., Modesti, M., Altmeyer, M., Lopes, M., 2020. Sequential role of RAD51 paralog complexes in replication fork remodeling and restart. Nat Commun 11, 3531. https://doi.org/10.1038/s41467-020-17324-z

Bonilla, B., Hengel, S.R., Grundy, M.K., Bernstein, K.A., 2020. RAD51 Gene Family Structure and Function. Annu Rev Genet 54, 25-46. https://doi.org/10.1146/annurev-genet-021920-092410

Bradbury, A., Hall, S., Curtin, N., Drew, Y., 2020. Targeting ATR as Cancer Therapy: A new era for synthetic lethality and synergistic combinations? Pharmacol Ther 207, 107450. https://doi.org/10.1016/j.pharmthera.2019.107450

Brinkman, E.K., Chen, T., Amendola, M., van Steensel, B., 2014. Easy quantitative assessment of genome editing by sequence trace decomposition. Nucleic Acids Res 42, e168. https://doi.org/10.1093/nar/gku936

Brown, J.S., O'Carrigan, B., Jackson, S.P., Yap, T.A., 2017. Targeting DNA Repair in Cancer: Beyond PARP Inhibitors. Cancer Discov 7, 20-37. https://doi.org/10.1158/2159-8290.CD-16-0860

Bryant, H.E., Schultz, N., Thomas, H.D., Parker, K.M., Flower, D., Lopez, E., Kyle, S., Meuth, M., Curtin, N.J., Helleday, T., 2005. Specific killing of BRCA2-deficient tumours with inhibitors of poly(ADP-ribose) polymerase. Nature 434, 913-917. https://doi.org/10.1038/nature03443

Cancer Genome Atlas Research Network, Weinstein, J.N., Collisson, E.A., Mills, G.B., Shaw, K.R.M., Ozenberger, B.A., Ellrott, K., Shmulevich, I., Sander, C., Stuart, J.M., 2013. The Cancer Genome Atlas Pan-Cancer analysis project. Nat Genet 45, 1113-1120. https://doi.org/10.1038/ng.2764

Cong, K., Peng, M., Kousholt, A.N., Lee, W.T.C., Lee, S., Nayak, S., Krais, J., VanderVere-Carozza, P.S., Pawelczak, K.S., Calvo, J., Panzarino, N.J., Turchi, 
J.J., Johnson, N., Jonkers, J., Rothenberg, E., Cantor, S.B., 2021. Replication gaps are a key determinant of PARP inhibitor synthetic lethality with BRCA deficiency. Mol Cell 81, 3227. https://doi.org/10.1016/j.molcel.2021.07.015 de Bono, J., Ramanathan, R.K., Mina, L., Chugh, R., Glaspy, J., Rafii, S., Kaye, S., Sachdev, J., Heymach, J., Smith, D.C., Henshaw, J.W., Herriott, A., Patterson, M., Curtin, N.J., Byers, L.A., Wainberg, Z.A., 2017. Phase I, Dose-Escalation, Two-Part Trial of the PARP Inhibitor Talazoparib in Patients with Advanced Germline BRCA1/2 Mutations and Selected Sporadic Cancers. Cancer Discov 7, 620-629. https://doi.org/10.1158/2159-8290.CD-16-1250

Deeg, K.I., Chung, I., Bauer, C., Rippe, K., 2016. Cancer Cells with Alternative Lengthening of Telomeres Do Not Display a General Hypersensitivity to ATR Inhibition. Front Oncol 6, 186. https://doi.org/10.3389/fonc.2016.00186

Fagan-Solis, K.D., Simpson, D.A., Kumar, R.J., Martelotto, L.G., Mose, L.E., Rashid, N.U., Ho, A.Y., Powell, S.N., Wen, Y.H., Parker, J.S., Reis-Filho, J.S., Petrini, J.H.J., Gupta, G.P., 2020. A P53-Independent DNA Damage Response Suppresses Oncogenic Proliferation and Genome Instability. Cell Rep 30, 13851399.e7. https://doi.org/10.1016/j.celrep.2020.01.020

Fang, Y., McGrail, D.J., Sun, C., Labrie, M., Chen, X., Zhang, D., Ju, Z., Vellano, C.P., Lu, Y., Li, Y., Jeong, K.J., Ding, Z., Liang, J., Wang, S.W., Dai, H., Lee, S., Sahni, N., Mercado-Uribe, I., Kim, T.-B., Chen, K., Lin, S.-Y., Peng, G., Westin, S.N., Liu, J., O'Connor, M.J., Yap, T.A., Mills, G.B., 2019. Sequential Therapy with PARP and WEE1 Inhibitors Minimizes Toxicity while Maintaining Efficacy. Cancer Cell 35, 851-867.e7. https://doi.org/10.1016/j.ccell.2019.05.001

Farmer, H., McCabe, N., Lord, C.J., Tutt, A.N.J., Johnson, D.A., Richardson, T.B., Santarosa, M., Dillon, K.J., Hickson, I., Knights, C., Martin, N.M.B., Jackson, S.P., Smith, G.C.M., Ashworth, A., 2005. Targeting the DNA repair defect in BRCA mutant cells as a therapeutic strategy. Nature 434, 917-921. https://doi.org/10.1038/nature03445

Flynn, R.L., Cox, K.E., Jeitany, M., Wakimoto, H., Bryll, A.R., Ganem, N.J., Bersani, F., Pineda, J.R., Suvà, M.L., Benes, C.H., Haber, D.A., Boussin, F.D., Zou, L., 2015. Alternative lengthening of telomeres renders cancer cells hypersensitive to ATR inhibitors. Science 347, 273-277. https://doi.org/10.1126/science.1257216

Frei, E., Karon, M., Levin, R.H., Freireich, E.J., Taylor, R.J., Hananian, J., Selawry, O., Holland, J.F., Hoogstraten, B., Wolman, I.J., Abir, E., Sawitsky, A., Lee, S., Mills, S.D., Burgert, E.O., Spurr, C.L., Patterson, R.B., Ebaugh, F.G., James, G.W., Moon, J.H., 1965. The effectiveness of combinations of antileukemic agents in inducing and maintaining remission in children with acute leukemia. Blood 26, 642-656.

Garbarino, J., Eckroate, J., Sundaram, R.K., Jensen, R.B., Bindra, R.S., 2021. Loss of ATRX confers DNA repair defects and PARP inhibitor sensitivity. Transl Oncol 14, 101147. https://doi.org/10.1016/j.tranon.2021.101147

García, H.D., Bei, Y., von Stebut, J., Ibáñez, G., Imami, K., Gürgen, D., Rolff, J., Helmsauer, K., Timme, N., Bardinet, V., González, R.C., MacArthur, I.C., Pusch, F., Chen, C.Y., Schulz, J., Wengner, A.M., Furth, C., Lala, B., Eggert, A., Seifert, G., Hundsoerfer, P., Kirchner, M., Mertins, P., Selbach, M., Lissat, A., Schulte, J.H., Haase, K., Scheer, M., Ortiz, M.V., Henssen, A.G., 2020. Exploiting a 
PAX3-FOXO1-induced synthetic lethal ATR dependency for rhabdomyosarcoma therapy (preprint). Cancer Biology. https://doi.org/10.1101/2020.12.04.411413

Garcin, E.B., Gon, S., Sullivan, M.R., Brunette, G.J., Cian, A.D., Concordet, J.-P., Giovannangeli, C., Dirks, W.G., Eberth, S., Bernstein, K.A., Prakash, R., Jasin, M., Modesti, M., 2019. Differential Requirements for the RAD51 Paralogs in Genome Repair and Maintenance in Human Cells. PLoS Genet 15, e1008355. https://doi.org/10.1371/journal.pgen.1008355

Golmard, L., Caux-Moncoutier, V., Davy, G., Al Ageeli, E., Poirot, B., Tirapo, C., Michaux, D., Barbaroux, C., d'Enghien, C.D., Nicolas, A., Castéra, L., SastreGarau, X., Stern, M.-H., Houdayer, C., Stoppa-Lyonnet, D., 2013. Germline mutation in the RAD51B gene confers predisposition to breast cancer. BMC Cancer 13, 484. https://doi.org/10.1186/1471-2407-13-484

Hart, T., Tong, A.H.Y., Chan, K., Van Leeuwen, J., Seetharaman, A., Aregger, M., Chandrashekhar, M., Hustedt, N., Seth, S., Noonan, A., Habsid, A., Sizova, O., Nedyalkova, L., Climie, R., Tworzyanski, L., Lawson, K., Sartori, M.A., Alibeh, S., Tieu, D., Masud, S., Mero, P., Weiss, A., Brown, K.R., Usaj, M., Billmann, M., Rahman, M., Constanzo, M., Myers, C.L., Andrews, B.J., Boone, C., Durocher, D., Moffat, J., 2017. Evaluation and Design of Genome-Wide CRISPR/SpCas9 Knockout Screens. G3 (Bethesda) 7, 2719-2727. https://doi.org/10.1534/g3.117.041277

Herman, S.E.M., Wiestner, A., 2016. Preclinical modeling of novel therapeutics in chronic lymphocytic leukemia: the tools of the trade. Semin Oncol 43, 222-232. https://doi.org/10.1053/j.seminoncol.2016.02.007

Hsiau, T., Conant, D., Rossi, N., Maures, T., Waite, K., Yang, J., Joshi, S., Kelso, R., Holden, K., Enzmann, B.L., Stoner, R., 2018. Inference of CRISPR Edits from Sanger Trace Data (preprint). Bioinformatics. https://doi.org/10.1101/251082

Hussain, M., Mateo, J., Fizazi, K., Saad, F., Shore, N., Sandhu, S., Chi, K.N., Sartor, O., Agarwal, N., Olmos, D., Thiery-Vuillemin, A., Twardowski, P., Roubaud, G., Özgüroğlu, M., Kang, J., Burgents, J., Gresty, C., Corcoran, C., Adelman, C.A., de Bono, J., PROfound Trial Investigators, 2020. Survival with Olaparib in Metastatic Castration-Resistant Prostate Cancer. N Engl J Med 383, 2345-2357. https://doi.org/10.1056/NEJMoa2022485

Hustedt, N., Álvarez-Quilón, A., McEwan, A., Yuan, J.Y., Cho, T., Koob, L., Hart, T., Durocher, D., 2019. A consensus set of genetic vulnerabilities to ATR inhibition. Open Biol 9, 190156. https://doi.org/10.1098/rsob.190156

lanevski, A., Giri, A.K., Aittokallio, T., 2020. SynergyFinder 2.0: visual analytics of multidrug combination synergies. Nucleic Acids Res 48, W488-W493. https://doi.org/10.1093/nar/gkaa216

Jones, P., Wilcoxen, K., Rowley, M., Toniatti, C., 2015. Niraparib: A Poly(ADP-ribose) Polymerase (PARP) Inhibitor for the Treatment of Tumors with Defective Homologous Recombination. J Med Chem 58, 3302-3314. https://doi.org/10.1021/jm5018237

Kim, H., George, E., Ragland, R., Rafail, S., Zhang, R., Krepler, C., Morgan, M., Herlyn, M., Brown, E., Simpkins, F., 2017. Targeting the ATR/CHK1 Axis with PARP Inhibition Results in Tumor Regression in BRCA-Mutant Ovarian Cancer Models. 
Clin Cancer Res 23, 3097-3108. https://doi.org/10.1158/1078-0432.CCR-162273

Kim, H., Xu, H., George, E., Hallberg, D., Kumar, S., Jagannathan, V., Medvedev, S., Kinose, Y., Devins, K., Verma, P., Ly, K., Wang, Y., Greenberg, R.A., Schwartz, L., Johnson, N., Scharpf, R.B., Mills, G.B., Zhang, R., Velculescu, V.E., Brown, E.J., Simpkins, F., 2020. Combining PARP with ATR inhibition overcomes PARP inhibitor and platinum resistance in ovarian cancer models. Nat Commun 11, 3726. https://doi.org/10.1038/s41467-020-17127-2

Kwok, M., Davies, N., Agathanggelou, A., Smith, E., Oldreive, C., Petermann, E., Stewart, G., Brown, J., Lau, A., Pratt, G., Parry, H., Taylor, M., Moss, P., Hillmen, P., Stankovic, T., 2016. ATR inhibition induces synthetic lethality and overcomes chemoresistance in TP53- or ATM-defective chronic lymphocytic leukemia cells. Blood 127, 582-595. https://doi.org/10.1182/blood-2015-05-644872

Lecona, E., Fernandez-Capetillo, O., 2018. Targeting ATR in cancer. Nat Rev Cancer 18, 586-595. https://doi.org/10.1038/s41568-018-0034-3

Lloyd, R.L., Wijnhoven, P.W.G., Ramos-Montoya, A., Wilson, Z., Illuzzi, G., Falenta, K., Jones, G.N., James, N., Chabbert, C.D., Stott, J., Dean, E., Lau, A., Young, L.A., 2020. Combined PARP and ATR inhibition potentiates genome instability and cell death in ATM-deficient cancer cells. Oncogene 39, 4869-4883. https://doi.org/10.1038/s41388-020-1328-y

Loe, T.K., Li, J.S.Z., Zhang, Y., Azeroglu, B., Boddy, M.N., Denchi, E.L., 2020.

Telomere length heterogeneity in ALT cells is maintained by PML-dependent localization of the BTR complex to telomeres. Genes Dev 34, 650-662. https://doi.org/10.1101/gad.333963.119

Loveday, C., Turnbull, C., Ramsay, E., Hughes, D., Ruark, E., Frankum, J.R., Bowden, G., Kalmyrzaev, B., Warren-Perry, M., Snape, K., Adlard, J.W., Barwell, J., Berg, J., Brady, A.F., Brewer, C., Brice, G., Chapman, C., Cook, J., Davidson, R., Donaldson, A., Douglas, F., Greenhalgh, L., Henderson, A., Izatt, L., Kumar, A., Lalloo, F., Miedzybrodzka, Z., Morrison, P.J., Paterson, J., Porteous, M., Rogers, M.T., Shanley, S., Walker, L., Breast Cancer Susceptibility Collaboration (UK), Eccles, D., Evans, D.G., Renwick, A., Seal, S., Lord, C.J., Ashworth, A., ReisFilho, J.S., Antoniou, A.C., Rahman, N., 2011. Germline mutations in RAD51D confer susceptibility to ovarian cancer. Nat Genet 43, 879-882. https://doi.org/10.1038/ng.893

Lu, R., O'Rourke, J.J., Sobinoff, A.P., Allen, J.A.M., Nelson, C.B., Tomlinson, C.G., Lee, M., Reddel, R.R., Deans, A.J., Pickett, H.A., 2019. The FANCM-BLM-TOP3ARMI complex suppresses alternative lengthening of telomeres (ALT). Nat Commun 10, 2252. https://doi.org/10.1038/s41467-019-10180-6

Mi, H., Ebert, D., Muruganujan, A., Mills, C., Albou, L.-P., Mushayamaha, T., Thomas, P.D., 2021. PANTHER version 16: a revised family classification, tree-based classification tool, enhancer regions and extensive API. Nucleic Acids Research 49, D394-D403. https://doi.org/10.1093/nar/gkaa1106

Miao, chenkui, Tsujino, T., Takai, T., Fu, G., Tsutsumi, T., Sztupinszki, Z., Szallasi, Z., Mouw, K.W., Kibel, A.S., Jia, L., 2021. RB1 loss overrides PARP inhibitor sensitivity driven by RNASEH2B loss in prostate cancer (preprint). Cancer Biology. https://doi.org/10.1101/2021.11.08.467839 
Murai, J., Feng, Y., Yu, G.K., Ru, Y., Tang, S.-W., Shen, Y., Pommier, Y., 2016. Resistance to PARP inhibitors by SLFN11 inactivation can be overcome by ATR inhibition. Oncotarget 7, 76534-76550. https://doi.org/10.18632/oncotarget.12266

Murai, J., Huang, S.N., Das, B.B., Renaud, A., Zhang, Y., Doroshow, J.H., Ji, J., Takeda, S., Pommier, Y., 2012. Trapping of PARP1 and PARP2 by Clinical PARP Inhibitors. Cancer Res 72, 5588-5599. https://doi.org/10.1158/00085472.CAN-12-2753

Murai, J., Huang, S.-Y.N., Renaud, A., Zhang, Y., Ji, J., Takeda, S., Morris, J., Teicher, B., Doroshow, J.H., Pommier, Y., 2014. Stereospecific PARP trapping by BMN 673 and comparison with olaparib and rucaparib. Mol Cancer Ther 13, 433-443. https://doi.org/10.1158/1535-7163.MCT-13-0803

Noordermeer, S.M., Adam, S., Setiaputra, D., Barazas, M., Pettitt, S.J., Ling, A.K., Olivieri, M., Álvarez-Quilón, A., Moatti, N., Zimmermann, M., Annunziato, S., Krastev, D.B., Song, F., Brandsma, I., Frankum, J., Brough, R., Sherker, A., Landry, S., Szilard, R.K., Munro, M.M., McEwan, A., Goullet de Rugy, T., Lin, Z.Y., Hart, T., Moffat, J., Gingras, A.-C., Martin, A., van Attikum, H., Jonkers, J., Lord, C.J., Rottenberg, S., Durocher, D., 2018. The shieldin complex mediates 53BP1-dependent DNA repair. Nature 560, 117-121. https://doi.org/10.1038/s41586-018-0340-7

Nyquist, M.D., Corella, A., Coleman, I., De Sarkar, N., Kaipainen, A., Ha, G., Gulati, R., Ang, L., Chatterjee, P., Lucas, J., Pritchard, C., Risbridger, G., Isaacs, J., Montgomery, B., Morrissey, C., Corey, E., Nelson, P.S., 2020. Combined TP53 and RB1 Loss Promotes Prostate Cancer Resistance to a Spectrum of Therapeutics and Confers Vulnerability to Replication Stress. Cell Rep 31, 107669. https://doi.org/10.1016/j.celrep.2020.107669

Olivieri, M., Cho, T., Álvarez-Quilón, A., Li, K., Schellenberg, M.J., Zimmermann, M., Hustedt, N., Rossi, S.E., Adam, S., Melo, H., Heijink, A.M., Sastre-Moreno, G., Moatti, N., Szilard, R.K., McEwan, A., Ling, A.K., Serrano-Benitez, A., Ubhi, T., Feng, S., Pawling, J., Delgado-Sainz, I., Ferguson, M.W., Dennis, J.W., Brown, G.W., Cortés-Ledesma, F., Williams, R.S., Martin, A., Xu, D., Durocher, D., 2020. A Genetic Map of the Response to DNA Damage in Human Cells. Cell 182, 481 496.e21. https://doi.org/10.1016/j.cell.2020.05.040

Olivieri, M., Durocher, D., 2021. Genome-scale chemogenomic CRISPR screens in human cells using the TKOv3 library. STAR Protoc 2, 100321. https://doi.org/10.1016/j.xpro.2021.100321

Pilié, P.G., Tang, C., Mills, G.B., Yap, T.A., 2019. State-of-the-art strategies for targeting the DNA damage response in cancer. Nat Rev Clin Oncol 16, 81-104. https://doi.org/10.1038/s41571-018-0114-z

Pizzi, S., Sertic, S., Orcesi, S., Cereda, C., Bianchi, M., Jackson, A.P., Lazzaro, F., Plevani, P., Muzi-Falconi, M., 2015. Reduction of hRNase H2 activity in AicardiGoutières syndrome cells leads to replication stress and genome instability. Hum Mol Genet 24, 649-658. https://doi.org/10.1093/hmg/ddu485

Poole, L.A., Zhao, R., Glick, G.G., Lovejoy, C.A., Eischen, C.M., Cortez, D., 2015. SMARCAL1 maintains telomere integrity during DNA replication. Proc Natl Acad Sci U S A 112, 14864-14869. https://doi.org/10.1073/pnas.1510750112 
Pujade-Lauraine, E., Ledermann, J.A., Selle, F., Gebski, V., Penson, R.T., Oza, A.M., Korach, J., Huzarski, T., Poveda, A., Pignata, S., Friedlander, M., Colombo, N., Harter, P., Fujiwara, K., Ray-Coquard, I., Banerjee, S., Liu, J., Lowe, E.S., Bloomfield, R., Pautier, P., SOLO2/ENGOT-Ov21 investigators, 2017. Olaparib tablets as maintenance therapy in patients with platinum-sensitive, relapsed ovarian cancer and a BRCA1/2 mutation (SOLO2/ENGOT-Ov21): a double-blind, randomised, placebo-controlled, phase 3 trial. Lancet Oncol 18, 1274-1284. https://doi.org/10.1016/S1470-2045(17)30469-2

Reijns, M.A.M., Bubeck, D., Gibson, L.C.D., Graham, S.C., Baillie, G.S., Jones, E.Y., Jackson, A.P., 2011. The structure of the human RNase $\mathrm{H} 2$ complex defines key interaction interfaces relevant to enzyme function and human disease. J Biol Chem 286, 10530-10539. https://doi.org/10.1074/jbc.M110.177394

Reijns, M.A.M., Jackson, A.P., 2014. Ribonuclease H2 in health and disease. Biochem Soc Trans 42, 717-725. https://doi.org/10.1042/BST20140079

Reijns, M.A.M., Rabe, B., Rigby, R.E., Mill, P., Astell, K.R., Lettice, L.A., Boyle, S., Leitch, A., Keighren, M., Kilanowski, F., Devenney, P.S., Sexton, D., Grimes, G., Holt, I.J., Hill, R.E., Taylor, M.S., Lawson, K.A., Dorin, J.R., Jackson, A.P., 2012. Enzymatic removal of ribonucleotides from DNA is essential for mammalian genome integrity and development. Cell 149, 1008-1022. https://doi.org/10.1016/j.cell.2012.04.011

Rein, H.L., Bernstein, K.A., Baldock, R.A., 2021. RAD51 paralog function in replicative DNA damage and tolerance. Curr Opin Genet Dev 71, 86-91. https://doi.org/10.1016/j.gde.2021.06.010

Robson, M., Goessl, C., Domchek, S., 2017. Olaparib for Metastatic Germline BRCAMutated Breast Cancer. N Engl J Med 377, 1792-1793. https://doi.org/10.1056/NEJMc1711644

Roulston, A., Zimmermann, M., Papp, R., Skeldon, A., Pellerin, C., Dumas-Berube, E., Dumais, V., Dorich, S., Fader, L.D., Fournier, S., Li, L., Leclaire, M-E., Yin, S.Y., Chefson, A., Alam, H., Yang, W., Fugere-Desjardins, C., Vignini-Hammond, S., Skorey, K., Mulani, A., Rimkunas, V., Veloso, A., Hamel, M., Stocco, R., Mamane, Y., Li, Z., Young, J., Zinda, M., Black, W.C. 2021. RP-3500: A Novel, Potent, and Selective ATR Inhibitor that is Effective in Preclinical Models as a Monotherapy and in Combination with PARP Inhibitors. Mol Cancer Therapeutics. In press.

Saldivar, J.C., Cortez, D., Cimprich, K.A., 2017. The essential kinase ATR: ensuring faithful duplication of a challenging genome. Nat Rev Mol Cell Biol 18, 622-636. https://doi.org/10.1038/nrm.2017.67

Saxena, S., Dixit, S., Somyajit, K., Nagaraju, G., 2019. ATR Signaling Uncouples the Role of RAD51 Paralogs in Homologous Recombination and Replication Stress Response. Cell Rep 29, 551-559.e4. https://doi.org/10.1016/j.celrep.2019.09.008

Schoonen, P.M., Kok, Y.P., Wierenga, E., Bakker, B., Foijer, F., Spierings, D.C.J., van Vugt, M.A.T.M., 2019. Premature mitotic entry induced by ATR inhibition potentiates olaparib inhibition-mediated genomic instability, inflammatory signaling, and cytotoxicity in BRCA2-deficient cancer cells. Mol Oncol 13, 24222440. https://doi.org/10.1002/1878-0261.12573

Setton, J., Zinda, M., Riaz, N., Durocher, D., Zimmermann, M., Koehler, M., Reis-Filho, J.S., Powell, S.N., 2021. Synthetic Lethality in Cancer Therapeutics: The Next 
Generation. Cancer Discov 11, 1626-1635. https://doi.org/10.1158/21598290.CD-20-1503

Shen, Y., Rehman, F.L., Feng, Y., Boshuizen, J., Bajrami, I., Elliott, R., Wang, B., Lord, C.J., Post, L.E., Ashworth, A., 2013. BMN 673, a novel and highly potent PARP1/2 inhibitor for the treatment of human cancers with DNA repair deficiency. Clin Cancer Res 19, 5003-5015. https://doi.org/10.1158/10780432.CCR-13-1391

Silva, B., Pentz, R., Figueira, A.M., Arora, R., Lee, Y.W., Hodson, C., Wischnewski, H., Deans, A.J., Azzalin, C.M., 2019. FANCM limits ALT activity by restricting telomeric replication stress induced by deregulated BLM and R-loops. Nat Commun 10, 2253. https://doi.org/10.1038/s41467-019-10179-z

Singh, T.R., Ali, A.M., Paramasivam, M., Pradhan, A., Wahengbam, K., Seidman, M.M., Meetei, A.R., 2013. ATR-dependent phosphorylation of FANCM at serine 1045 is essential for FANCM functions. Cancer Res 73, 4300-4310. https://doi.org/10.1158/0008-5472.CAN-12-3976

Somyajit, K., Saxena, S., Babu, S., Mishra, A., Nagaraju, G., 2020. Mammalian RAD51 paralogs protect nascent DNA at stalled forks and mediate replication restart. Nucleic Acids Res 48, 5196-5197. https://doi.org/10.1093/nar/gkaa279

Stewart, E., Goshorn, R., Bradley, C., Griffiths, L.M., Benavente, C., Twarog, N.R., Miller, G.M., Caufield, W., Freeman, B.B., Bahrami, A., Pappo, A., Wu, J., Loh, A., Karlström, Å., Calabrese, C., Gordon, B., Tsurkan, L., Hatfield, M.J., Potter, P.M., Snyder, S.E., Thiagarajan, S., Shirinifard, A., Sablauer, A., Shelat, A.A., Dyer, M.A., 2014. Targeting the DNA repair pathway in Ewing sarcoma. Cell Rep 9, 829-841. https://doi.org/10.1016/j.celrep.2014.09.028

Swisher, E.M., Kwan, T.T., Oza, A.M., Tinker, A.V., Ray-Coquard, I., Oaknin, A., Coleman, R.L., Aghajanian, C., Konecny, G.E., O'Malley, D.M., Leary, A., Provencher, D., Welch, S., Chen, L.-M., Wahner Hendrickson, A.E., Ma, L., Ghatage, P., Kristeleit, R.S., Dorigo, O., Musafer, A., Kaufmann, S.H., Elvin, J.A., Lin, D.I., Chambers, S.K., Dominy, E., Vo, L.-T., Goble, S., Maloney, L., Giordano, H., Harding, T., Dobrovic, A., Scott, C.L., Lin, K.K., McNeish, I.A., 2021. Molecular and clinical determinants of response and resistance to rucaparib for recurrent ovarian cancer treatment in ARIEL2 (Parts 1 and 2). Nat Commun 12, 2487. https://doi.org/10.1038/s41467-021-22582-6

Syed, A., Tainer, J.A., 2018. The MRE11-RAD50-NBS1 Complex Conducts the Orchestration of Damage Signaling and Outcomes to Stress in DNA Replication and Repair. Annu Rev Biochem 87, 263-294. https://doi.org/10.1146/annurevbiochem-062917-012415

Toledo, L.I., Altmeyer, M., Rask, M.-B., Lukas, C., Larsen, D.H., Povlsen, L.K., BekkerJensen, S., Mailand, N., Bartek, J., Lukas, J., 2013. ATR prohibits replication catastrophe by preventing global exhaustion of RPA. Cell 155, 1088-1103. https://doi.org/10.1016/j.cell.2013.10.043

Verma, P., Dilley, R.L., Zhang, T., Gyparaki, M.T., Li, Y., Greenberg, R.A., 2019. RAD52 and SLX4 act nonepistatically to ensure telomere stability during alternative telomere lengthening. Genes Dev 33, 221-235. https://doi.org/10.1101/gad.319723.118 
Wang, C., Wang, G., Feng, X., Shepherd, P., Zhang, J., Tang, M., Chen, Z., Srivastava, M., McLaughlin, M.E., Navone, N.M., Hart, G.T., Chen, J., 2019. Genome-wide CRISPR screens reveal synthetic lethality of RNASEH2 deficiency and ATR inhibition. Oncogene 38, 2451-2463. https://doi.org/10.1038/s41388-018-0606-4

Yadav, B., Wennerberg, K., Aittokallio, T., Tang, J., 2015. Searching for Drug Synergy in Complex Dose-Response Landscapes Using an Interaction Potency Model. Comput Struct Biotechnol J 13, 504-513. https://doi.org/10.1016/j.csbj.2015.09.001

Yap, T.A., Krebs, M.G., Postel-Vinay, S., Bang, Y.J., El-Khoueiry, A., Abida, W., Harrington, K., Sundar, R., Carter, L., Castanon-Alvarez, E., Im, S.A., Berges, A., Khan, M., Stephens, C., Ross, G., Soria, J.C., 2016. Phase I modular study of AZD6738, a novel oral, potent and selective ataxia telangiectasia Rad3-related (ATR) inhibitor in combination (combo) with carboplatin, olaparib or durvalumab in patients (pts) with advanced cancers. European Journal of Cancer 69, S2. https://doi.org/10.1016/S0959-8049(16)32607-7

Yazinski, S.A., Comaills, V., Buisson, R., Genois, M.-M., Nguyen, H.D., Ho, C.K., Todorova Kwan, T., Morris, R., Lauffer, S., Nussenzweig, A., Ramaswamy, S., Benes, C.H., Haber, D.A., Maheswaran, S., Birrer, M.J., Zou, L., 2017. ATR inhibition disrupts rewired homologous recombination and fork protection pathways in PARP inhibitor-resistant BRCA-deficient cancer cells. Genes Dev 31, 318-332. https://doi.org/10.1101/gad.290957.116

Zhang, J.-M., Yadav, T., Ouyang, J., Lan, L., Zou, L., 2019. Alternative Lengthening of Telomeres through Two Distinct Break-Induced Replication Pathways. Cell Rep 26, 955-968.e3. https://doi.org/10.1016/j.celrep.2018.12.102

Zhang, J.-M., Zou, L., 2020. Alternative lengthening of telomeres: from molecular mechanisms to therapeutic outlooks. Cell Biosci 10, 30. https://doi.org/10.1186/s13578-020-00391-6

Zimmermann, M., Murina, O., Reijns, M.A.M., Agathanggelou, A., Challis, R., Tarnauskaitè, Ž., Muir, M., Fluteau, A., Aregger, M., McEwan, A., Yuan, W., Clarke, M., Lambros, M.B., Paneesha, S., Moss, P., Chandrashekhar, M., Angers, S., Moffat, J., Brunton, V.G., Hart, T., de Bono, J., Stankovic, T., Jackson, A.P., Durocher, D., 2018. CRISPR screens identify genomic ribonucleotides as a source of PARP-trapping lesions. Nature 559, 285-289. https://doi.org/10.1038/s41586-018-0291-z 
Figure 1.

Zimmermann et al.

A

Day 0

TKOv3 sgRNA library

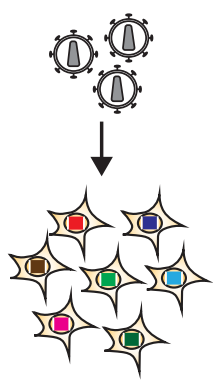

RPE1-hTERT

Cas9 TP53-KO

NGS quantification

of sgRNA representation
Day 6

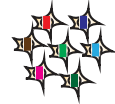

DMSO

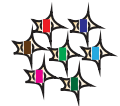

RP-3500 (ATRi) LD 20

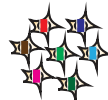

Niraparib (PARPi) $\mathrm{LD}_{20}$

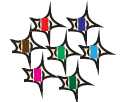

Combination $\mathrm{LD}_{20}$
Day 18
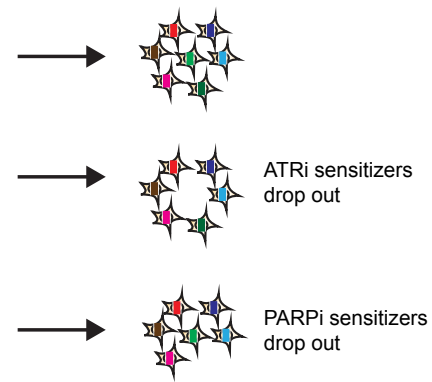

$\longrightarrow$ 迎 Combination sensitizers

NGS quantification

of sgRNA representation

Positive regulation of transcription of nucleolar large rRNA Interstrand cross-link repair

Positive regulation of double-strand break repair Reciprocal meiotic recombination Double-strand break repair via homologous recombination
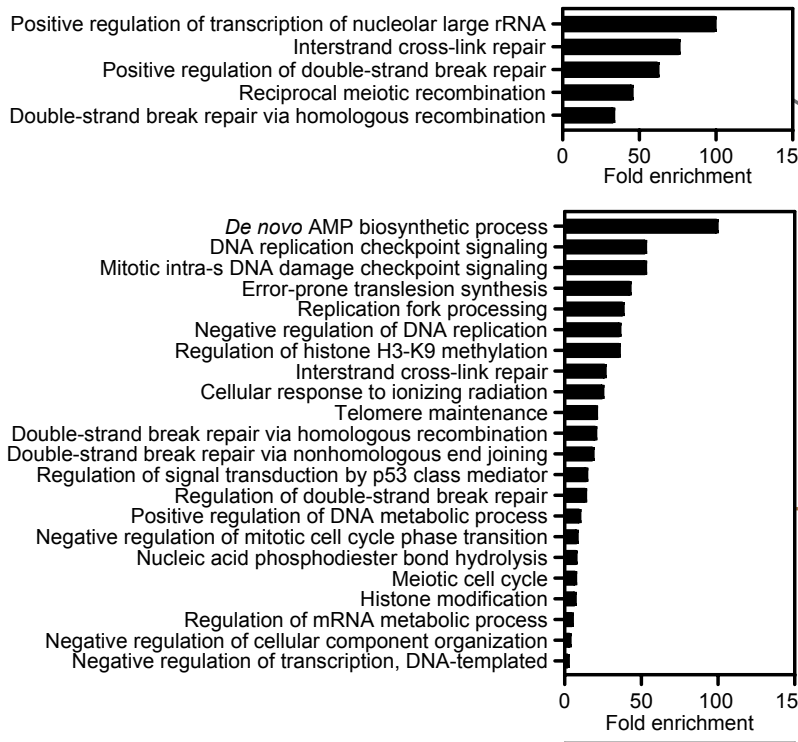

Positive regulation of telomerase catalytic core complex assembly

Establishment of protein-containing complex localization to telomere Establishment of RNA localization to telomere Negative regulation of protein ADP-ribosylation Negative regulation of telomere maintenance in response to DNA damage Meiotic cell cycle checkpoint signaling
Negative regulation of telomere capping Negative regulation of telomere capping Replication fork protection Telomere maintenance via recombination Protein localization to chromosome, telomeric region Mitotic intra-S DNA damage checkpoint signaling Regulation of cellular response to heat Replication fork processing

Double-strand break repair via nonhomologous end joining

Base-excision repair
Double-strand break repair via homologous recombination Cellular response to gamma radiation Mitotic G2 DNA damage checkpoint signaling Mitotic G2 DNA damage checkpoint signaling Positive regulation of telomere maintenance via telomerase Regulation of double-strand break repair via homologous recombination
Reciprocal meiotic recombination
DNA damage response, signal transduction by p53 class mediator

DNA damage response, signal transduction by p53 class mediator
Positive regulation of dna repair Positive regulation of mitotic cell cycle Regulation of nuclear division RNA catabolic process
Nucleic acid phosphodiester bond hydrolysis
Histone modification Histone modification
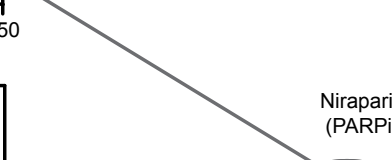
(2)

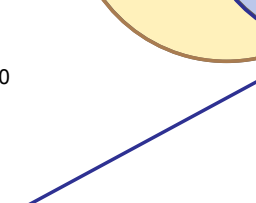

Combination 
Figure 2.

A

C

D

5637

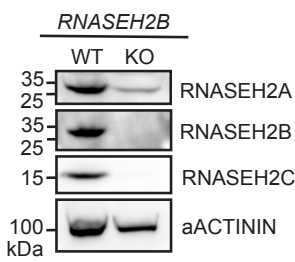

B

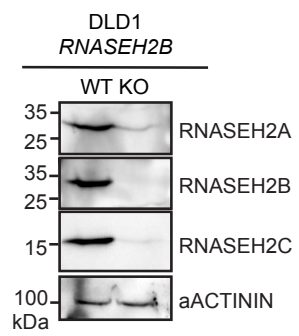

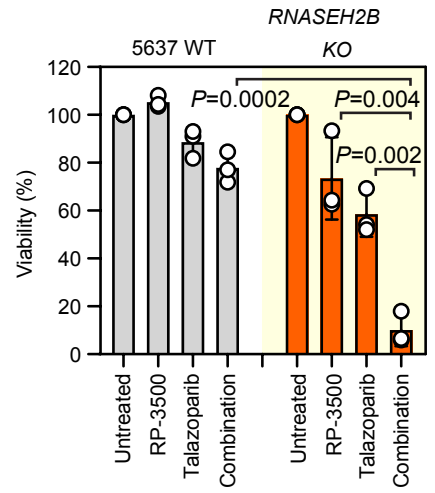

Talazoparib (PARPi): $3 \mathrm{nM}$ RP-3500 (ATRi): $5 \mathrm{nM}$

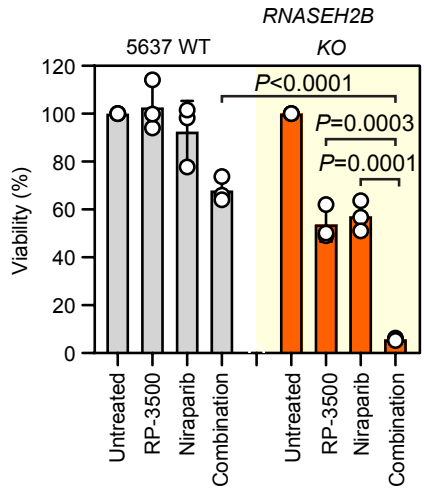

Niraparib (PARPi): $100 \mathrm{nM}$ RP-3500 (ATRi): 4 nM
E

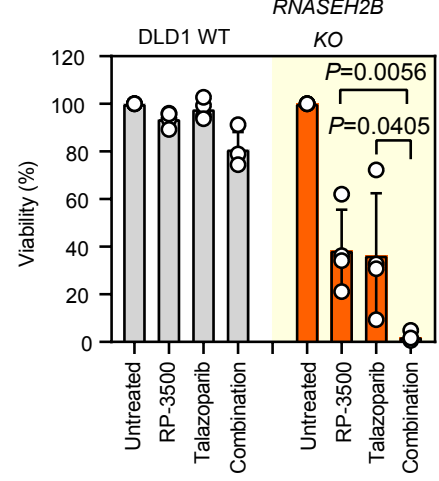

Talazoparib (PARPi): $2.5 \mathrm{nM}$ RP-3500 (ATRi): 7 nM
F

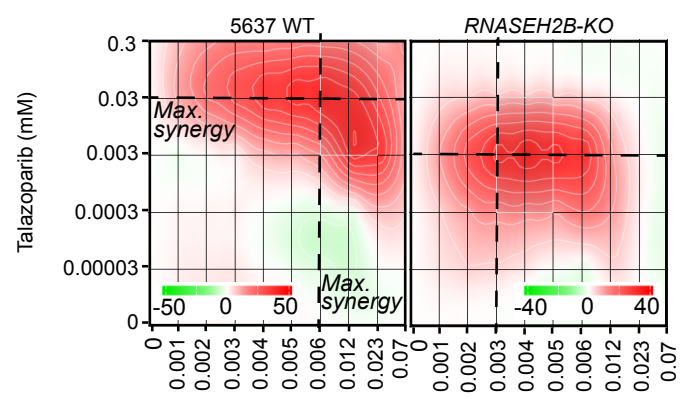

RP-3500 (mM)

H

ZIP synergy score

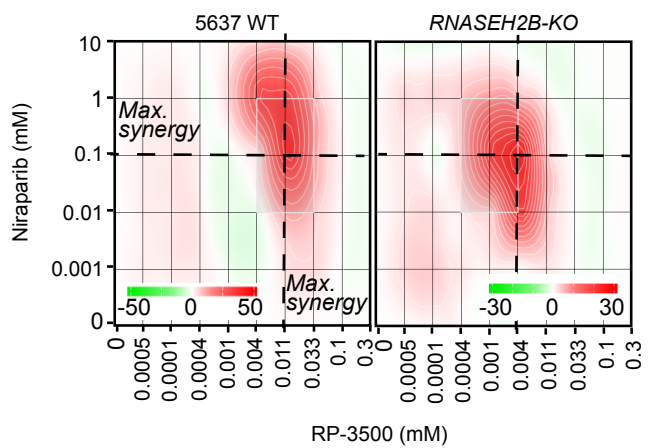

\section{ZIP synergy score}

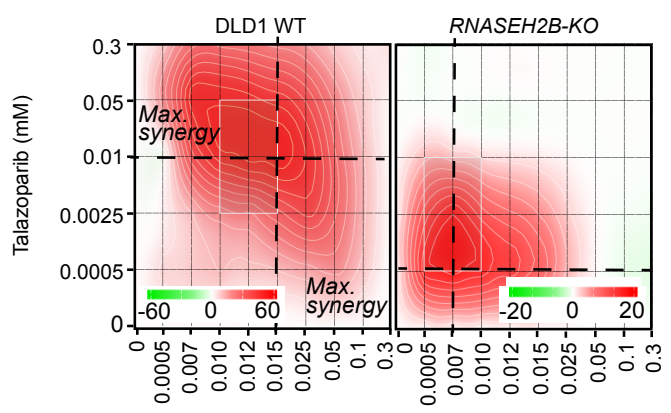

RP-3500 (mM) 
A

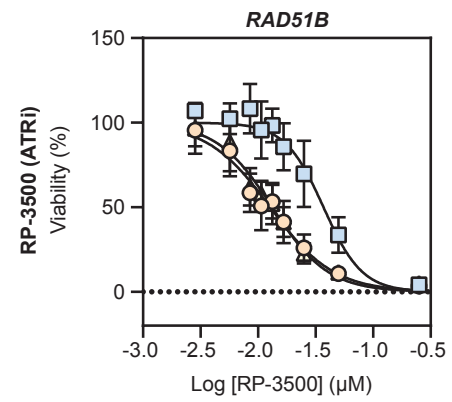

C

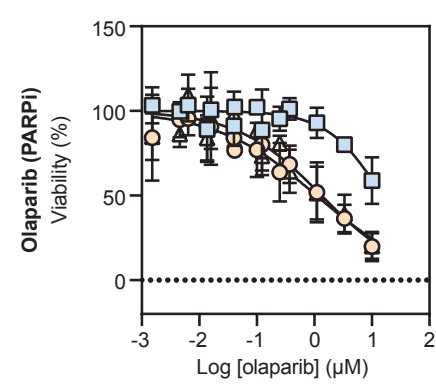

마 sgAAVS1

-O- sgRAD51B-1

$\triangle$ sgRAD51B-2
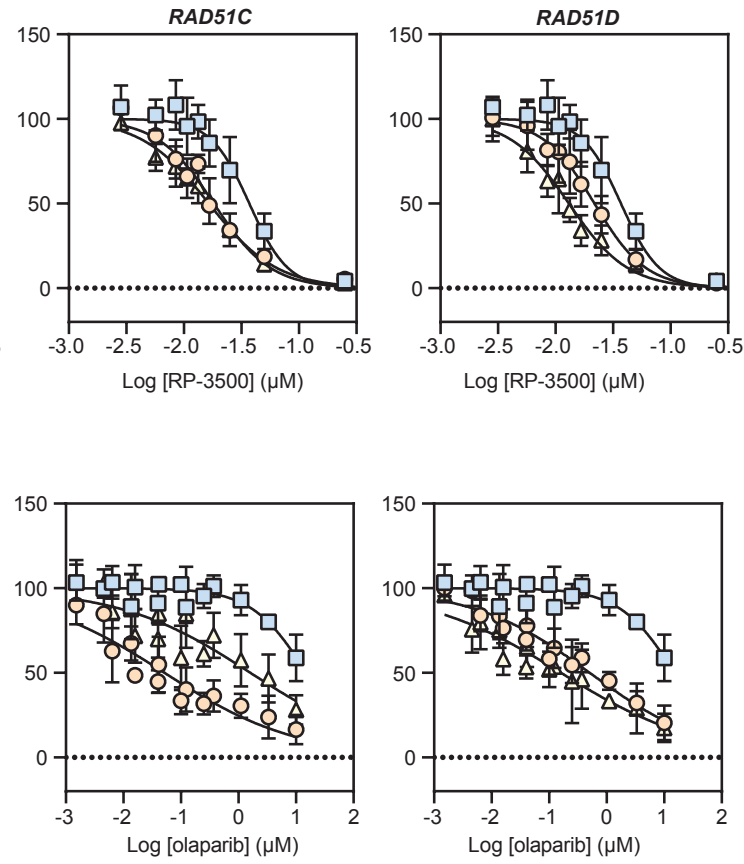

- sgAAVS1

-O- sgRAD51D-1

$\triangle$ sgRAD51D-2
B
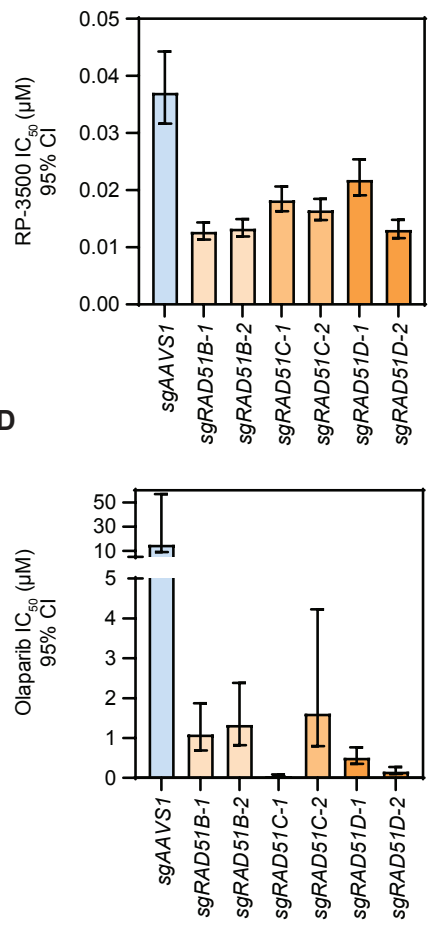
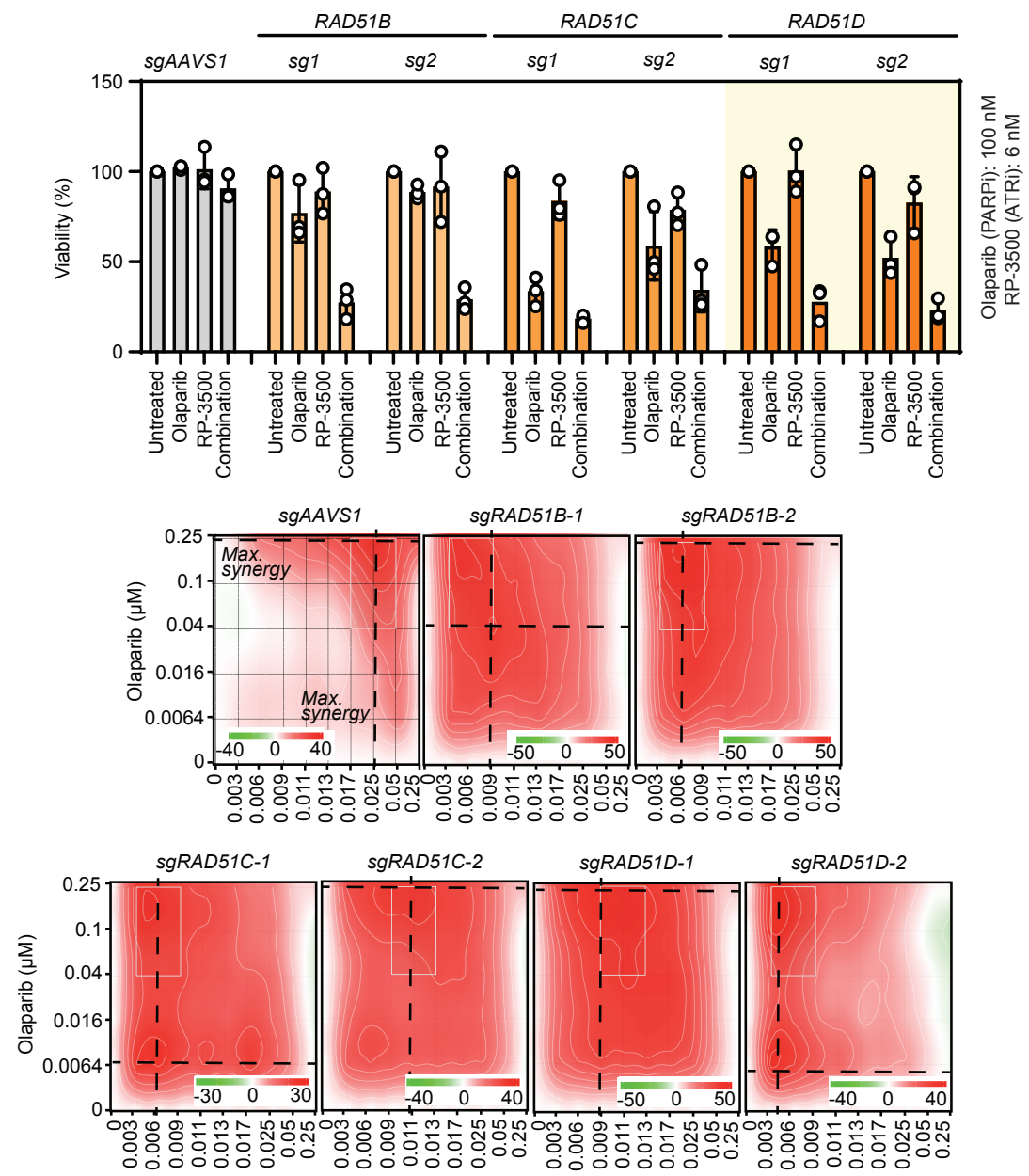
A

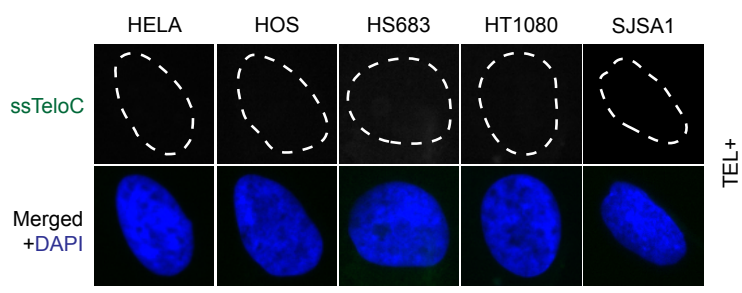

B
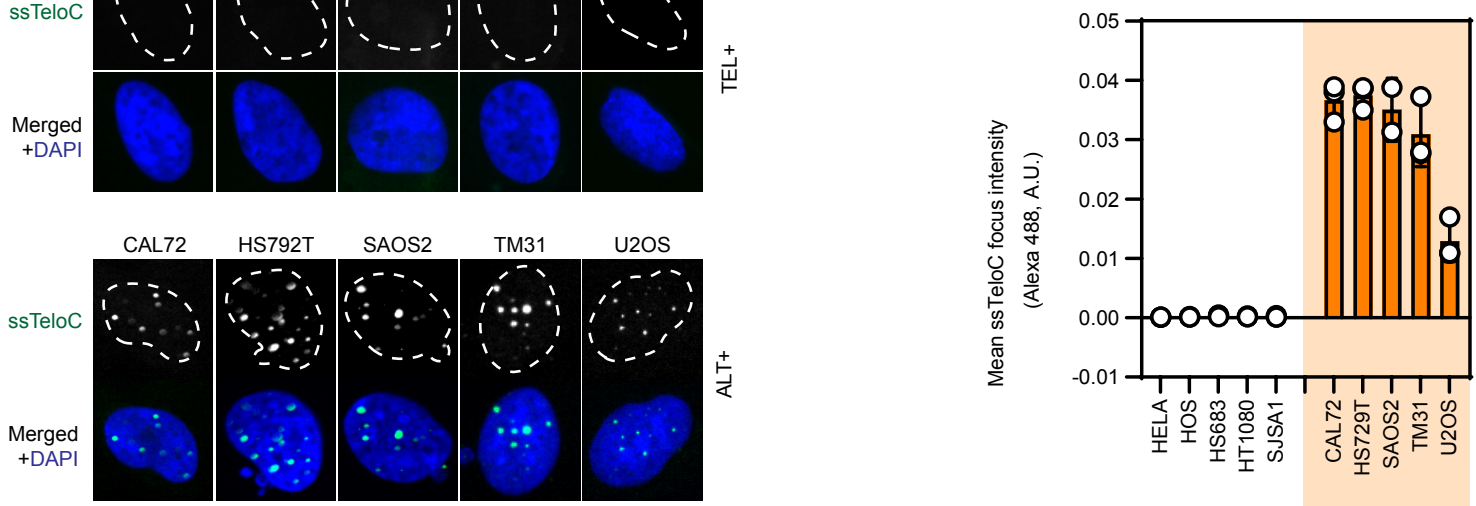

TEL+

ALT+
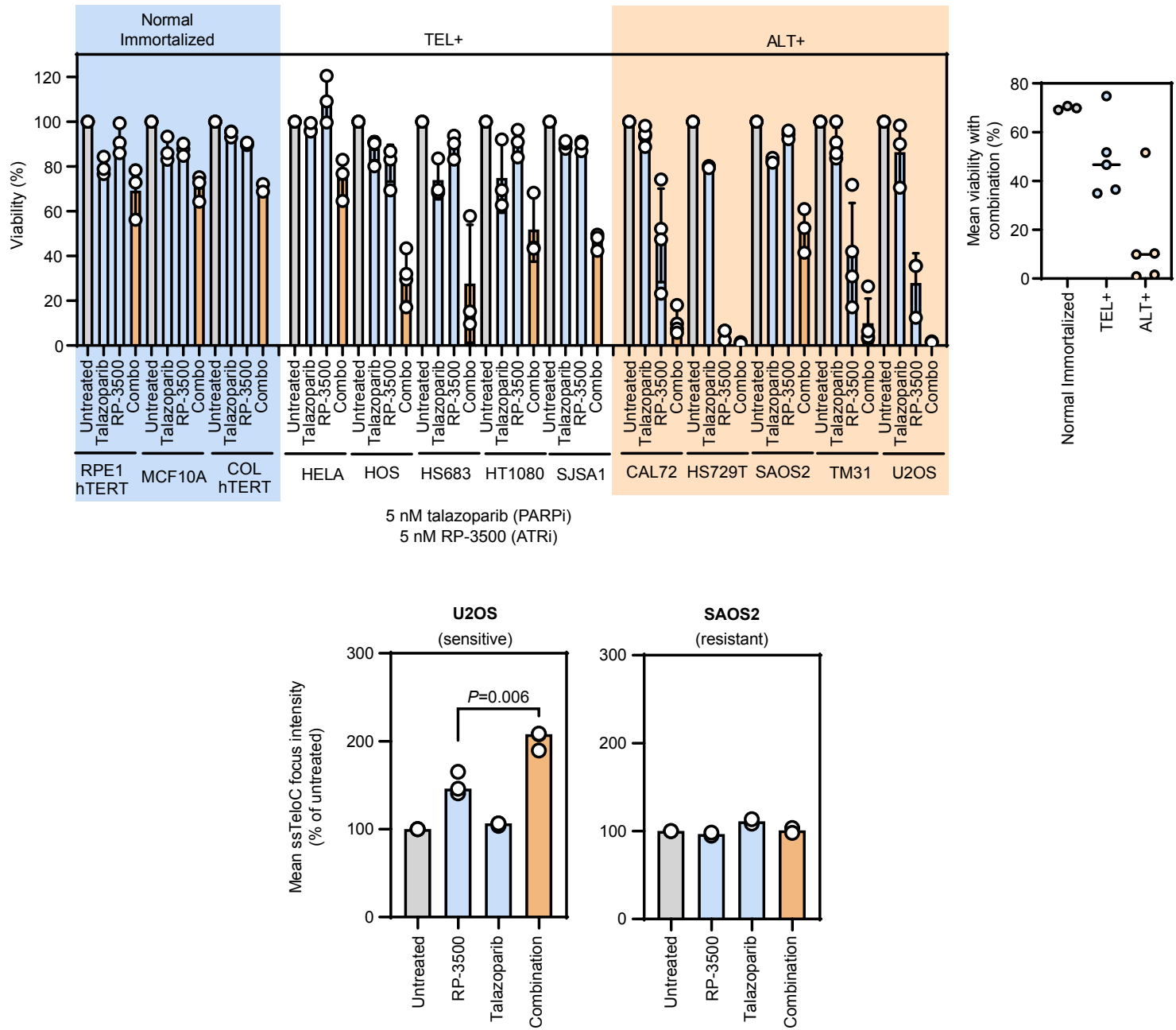
A

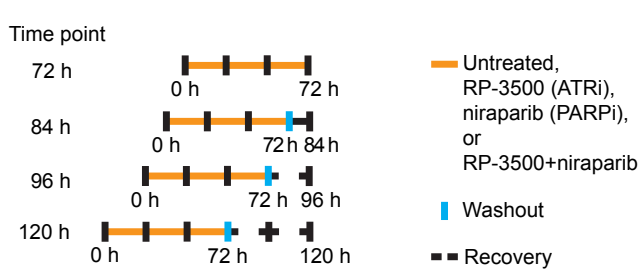

C

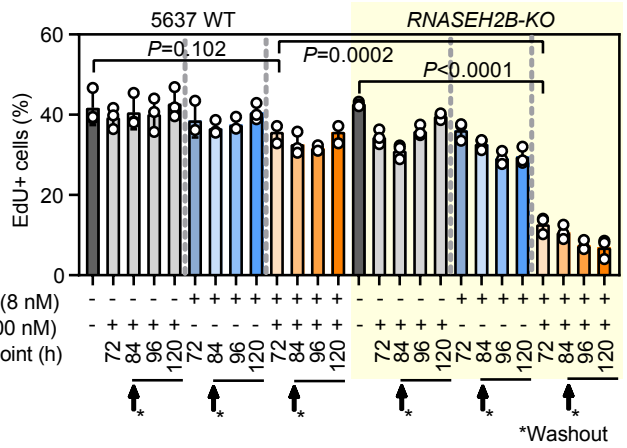

E

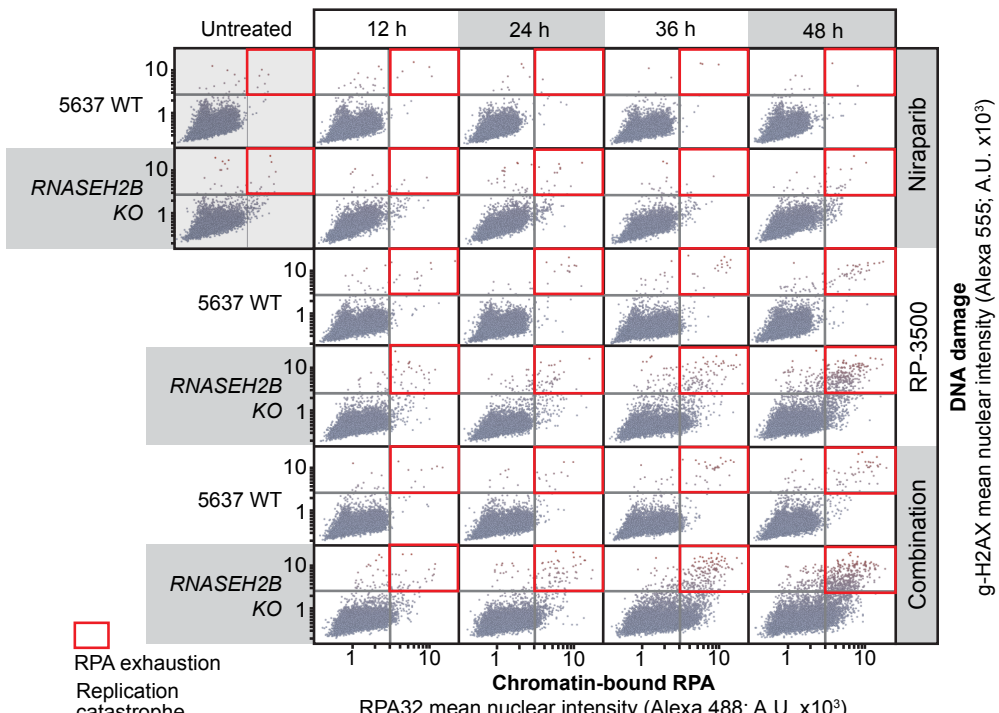

$\mathbf{F}$

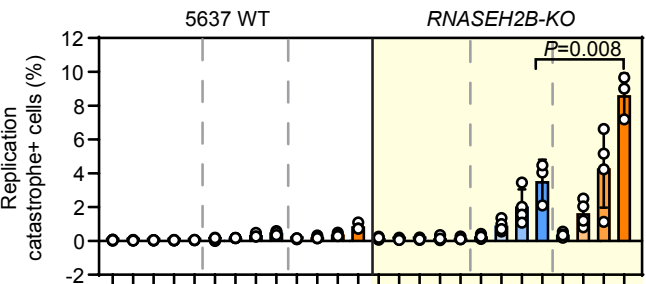

B

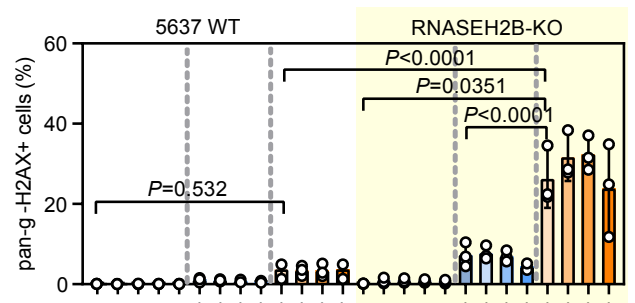

RP-3500 (8 nM)

Niraparib (200 nM)

Time point (h)

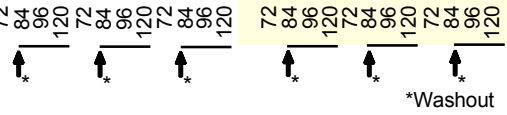

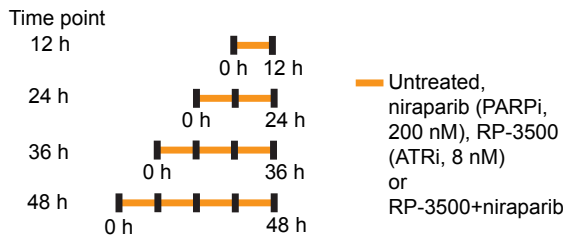

G

3 days on/ 4 days off

Continuous
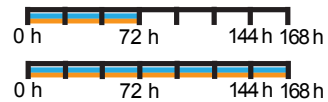

Continuous niraparib +

3 days on/4 days off RP-3500

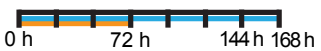

- RP-3500 (ATRi, 4 nM)

- Niraparib (PARPi, 100nM)

H

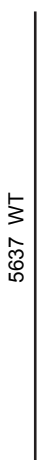



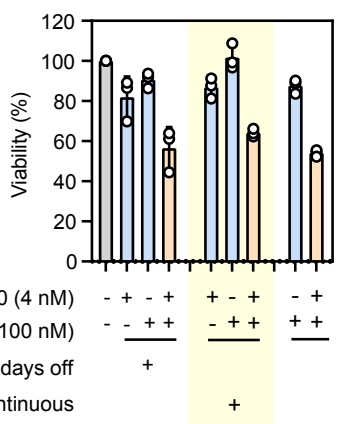

Continuous niraparib + 3 days on/ 4 days off

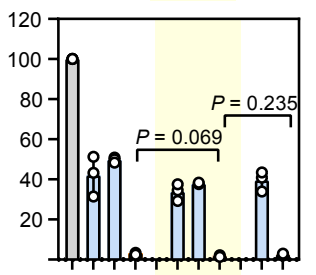

RP-3500 (4 nM) - + + + + + + Niraparib (100 nM) - $+++\underline{++}+++$ 3 days on/ 4 days off Continuous

Continuous niraparib + 3 days on/ 4 days off 
Figure 6.

Zimmermann et al.

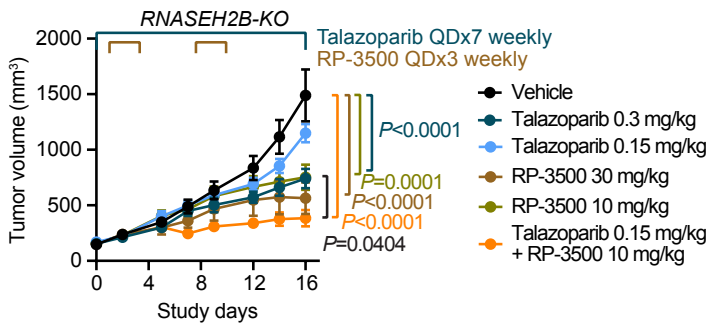

B

RNase H2-deficient TNBC PDX
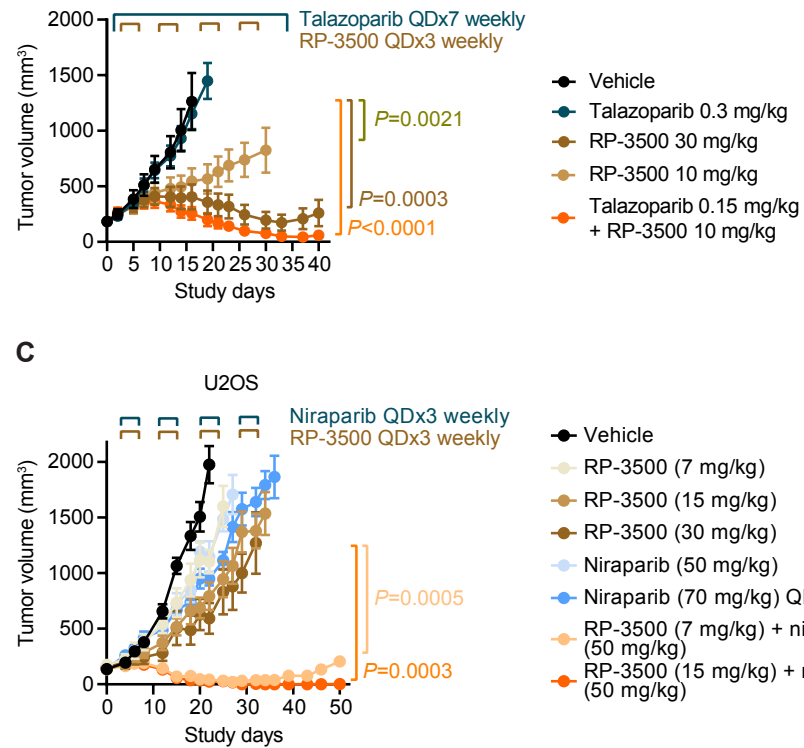
- Vehicle $\mathrm{RP}-3500$ (7 mg/kg)$$
\text { - RP-3500 (15 mg/kg) }
$$$$
\text { - RP-3500 (30 mg/kg) }
$$$$
\text { Niraparib }(50 \mathrm{mg} / \mathrm{kg})
$$$$
\text { - Niraparib (70 mg/kg) QDx5 }
$$$$
\text { RP-3500 (7 mg/kg) + niraparib }
$$$$
(50 \mathrm{mg} / \mathrm{kg})
$$$$
\text { RP-3500 (15 mg/kg) + niraparib }
$$$$
(50 \mathrm{mg} / \mathrm{kg} \text { ) }
$$

- Vehicle

Talazop RP-3500 $30 \mathrm{mg} / \mathrm{kg}$ $\mathrm{RP}-350010 \mathrm{mg} / \mathrm{kg}$ Talazoparib $0.15 \mathrm{mg} / \mathrm{kg}$ + RP-3500 $10 \mathrm{mg} / \mathrm{kg}$ 
bioRxiv preprint doi: https://doi.org/10.1101/2021.12.13.472393; this version posted December 14, 2021. The copyright holder for this preprint (which was not certified by peer review) is the author/funder. All rights reserved. No reuse allowed without permission.

\section{Supplementary Figure 1.}

a

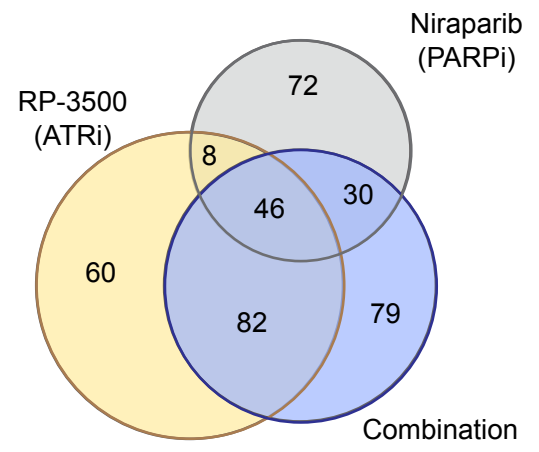

All hits $(P<0.05)$ b

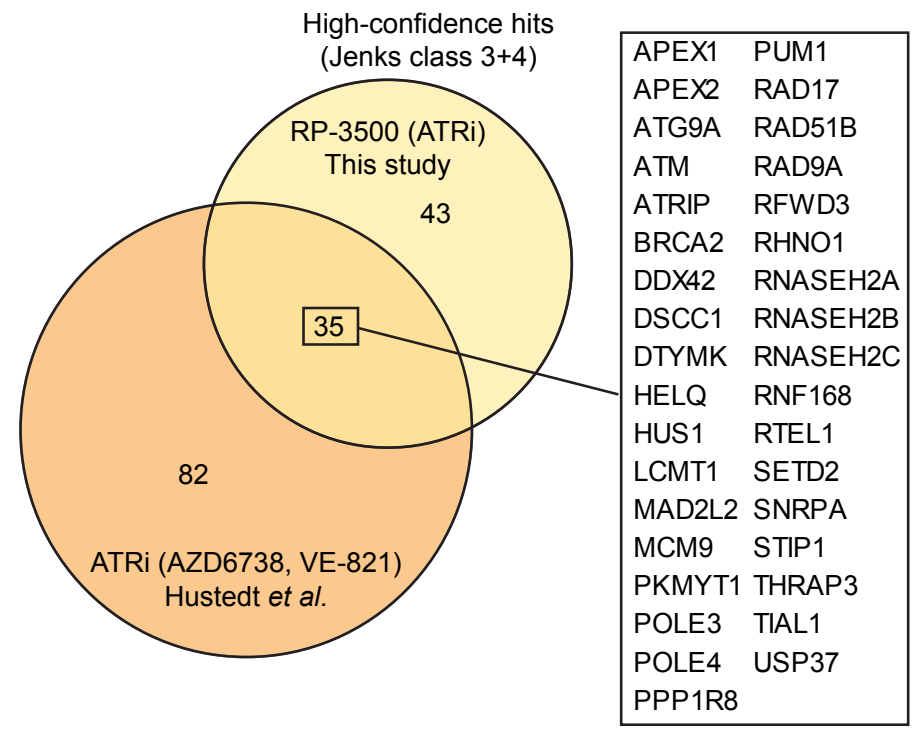

C
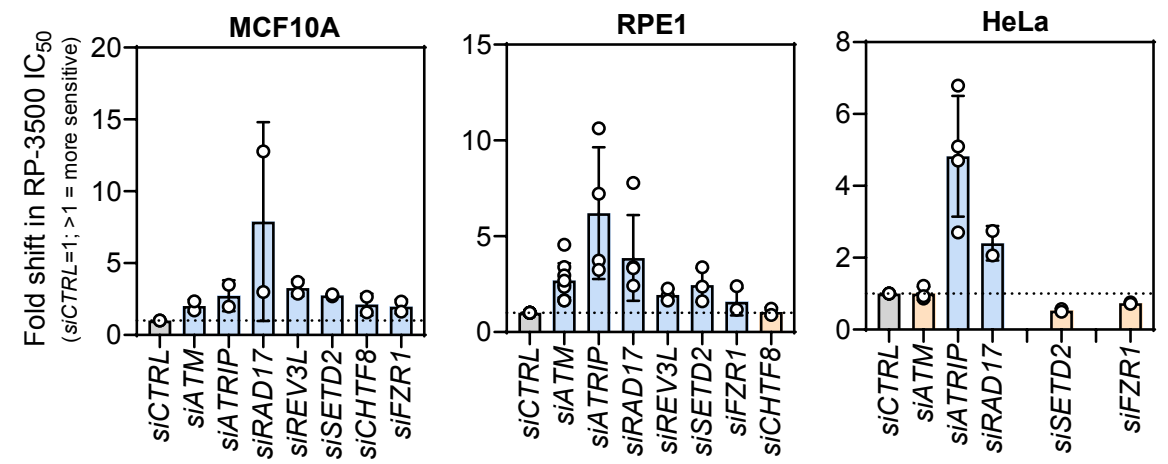

d

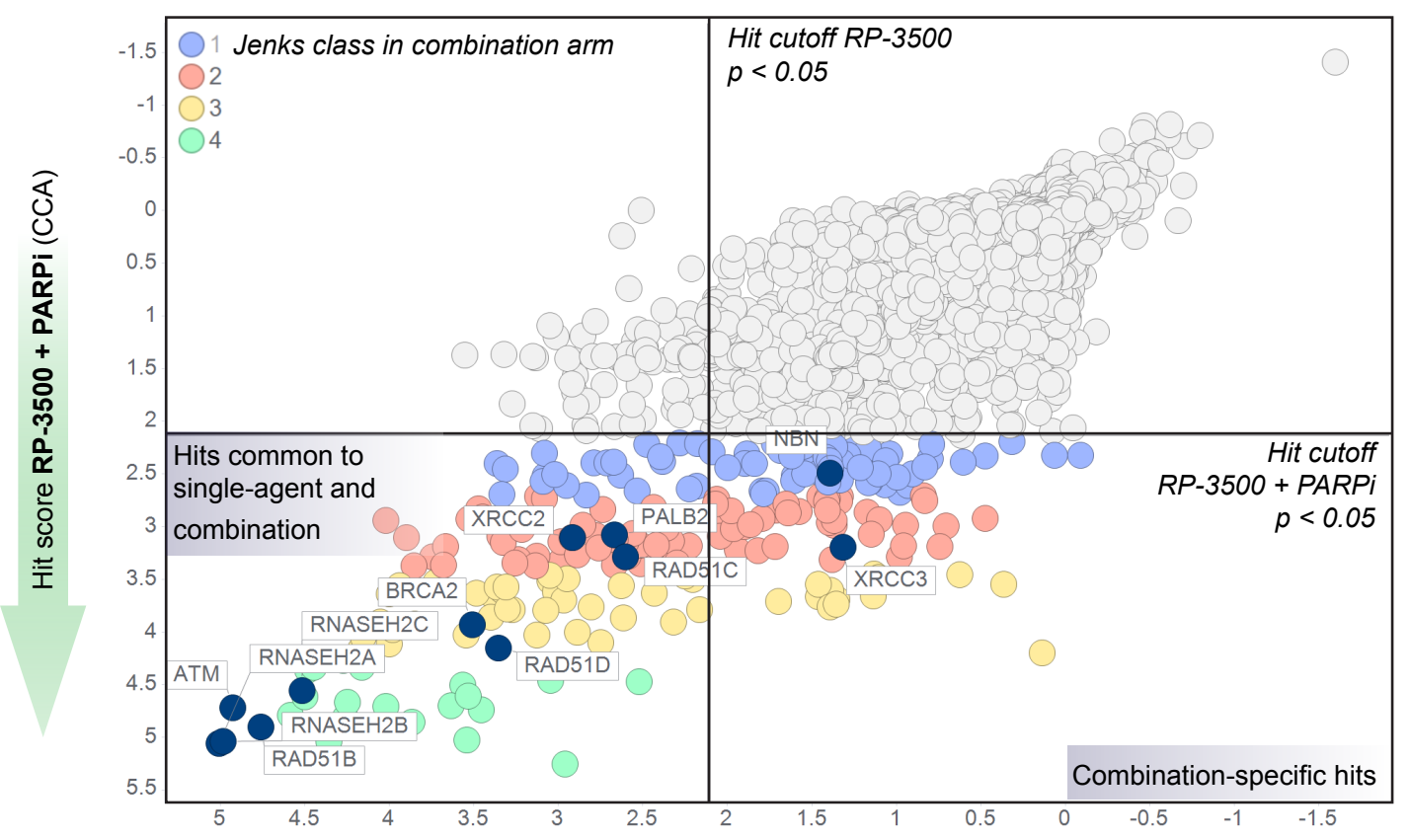


A

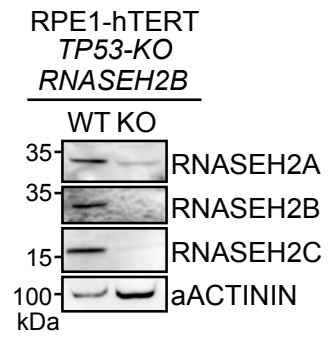

E

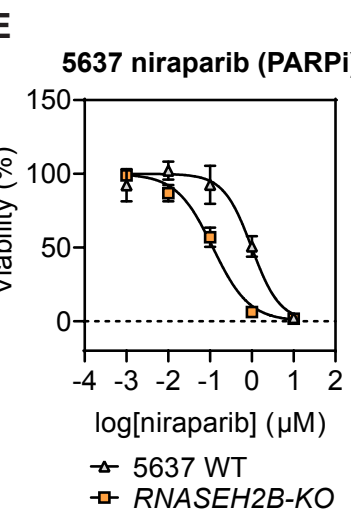

B

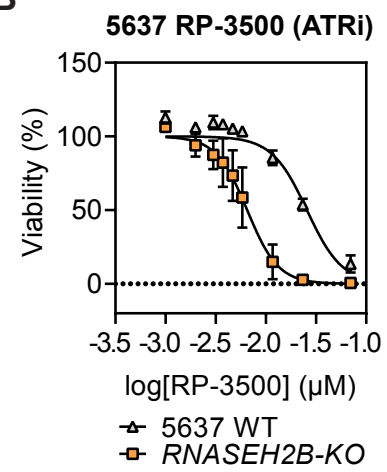

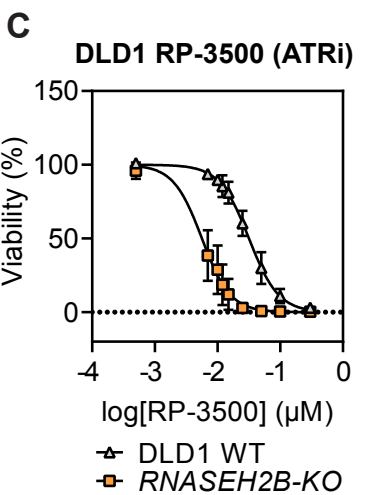

G
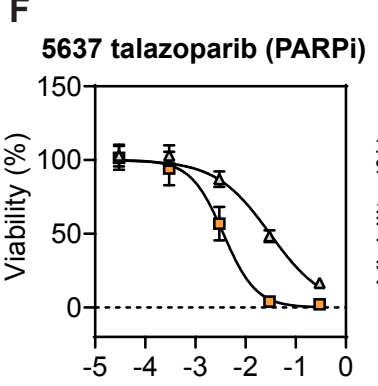

log [talazoparib] $(\mu \mathrm{M})$

$$
\begin{aligned}
& \triangle 5637 \text { WT } \\
& \rightarrow \text { RNASEH } 2 B-K O
\end{aligned}
$$

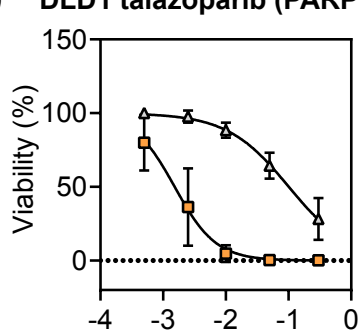

$\log$ [talazoparib] [ $\mu \mathrm{M}$ ]

$$
\begin{aligned}
& ₫ \text { DLD1 WT } \\
& \square \text { RNASEH2B-KO }
\end{aligned}
$$

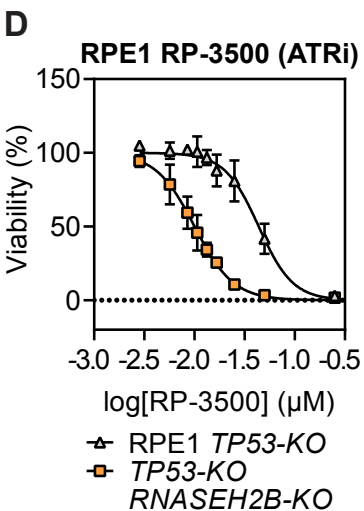

H

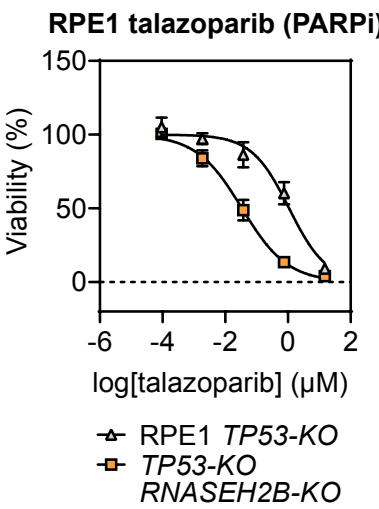

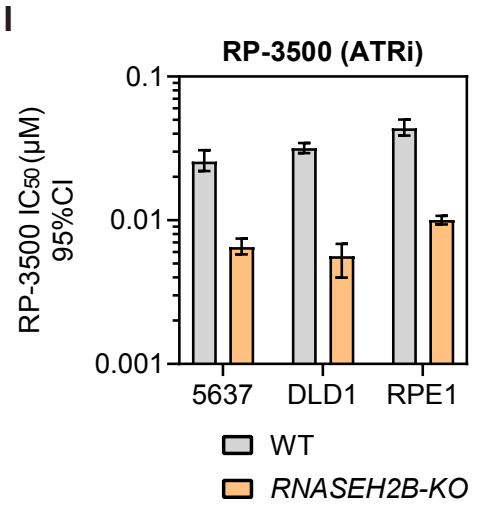

J

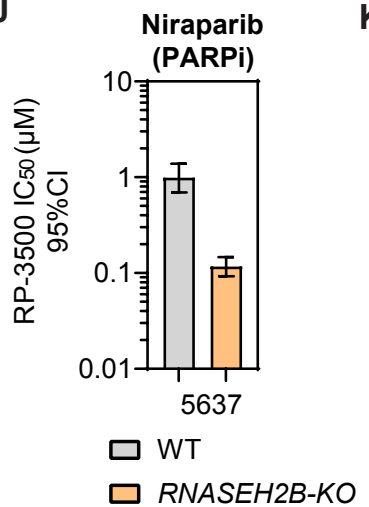

$\mathbf{K}$

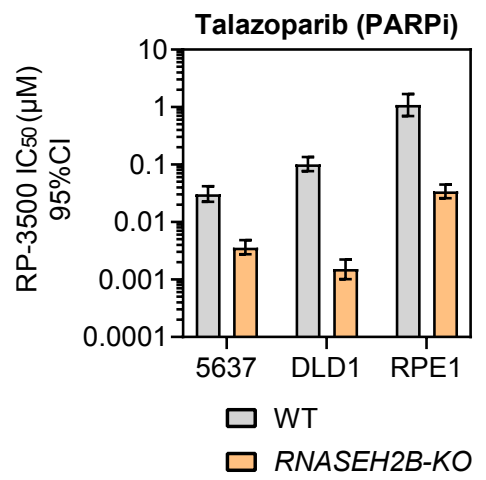


A

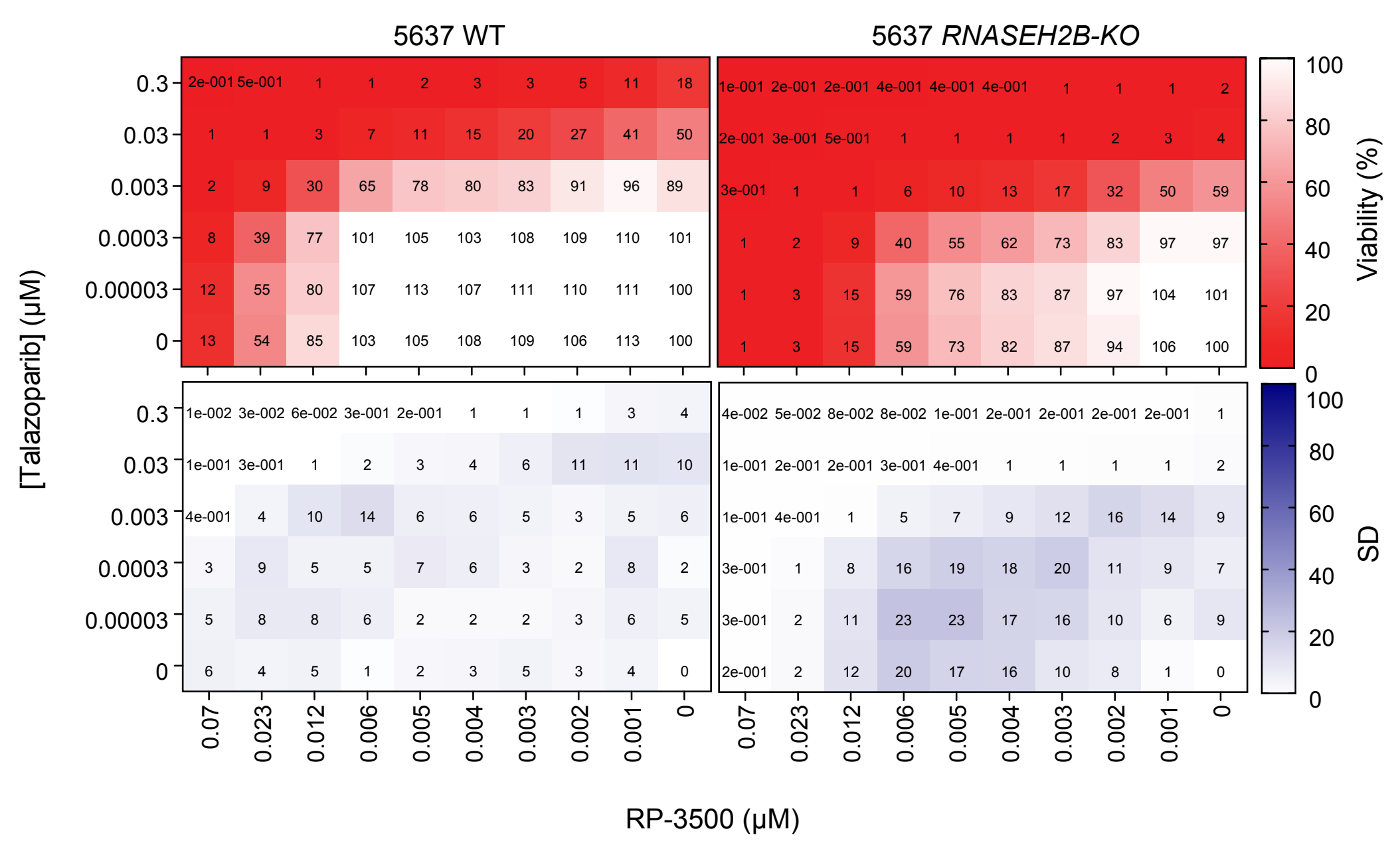

c

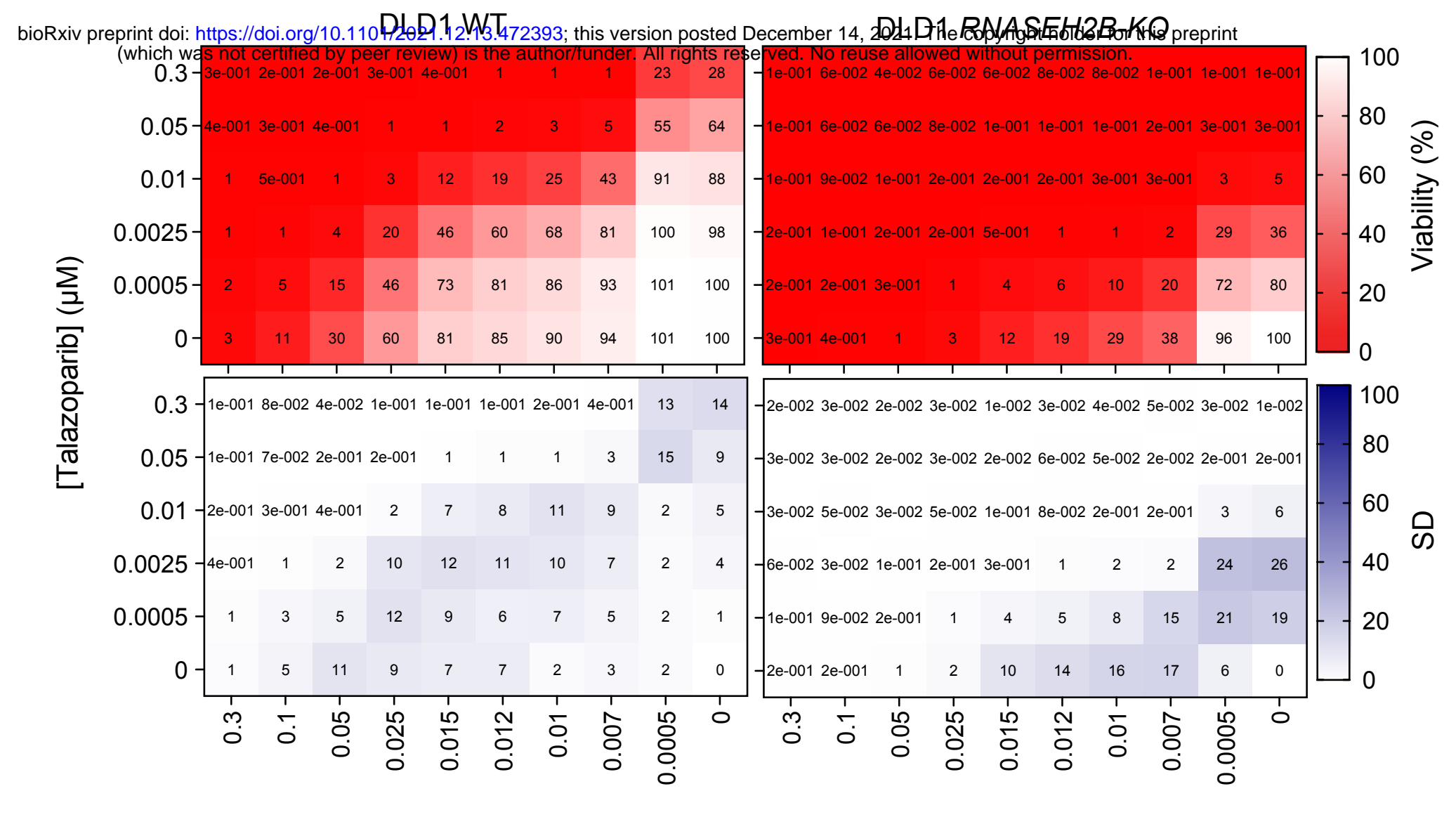

$\mathrm{RP}-3500(\mu \mathrm{M})$

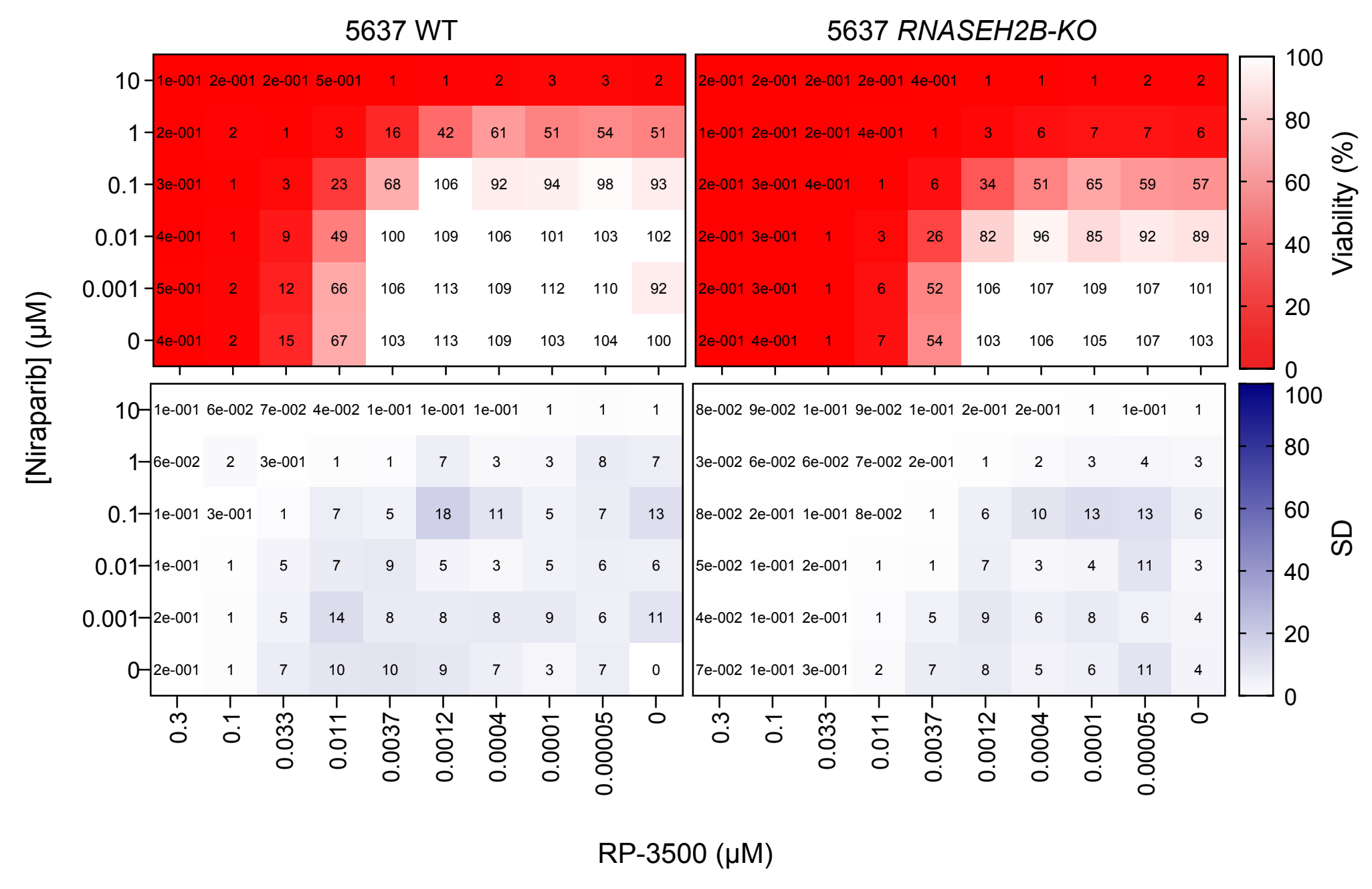

D

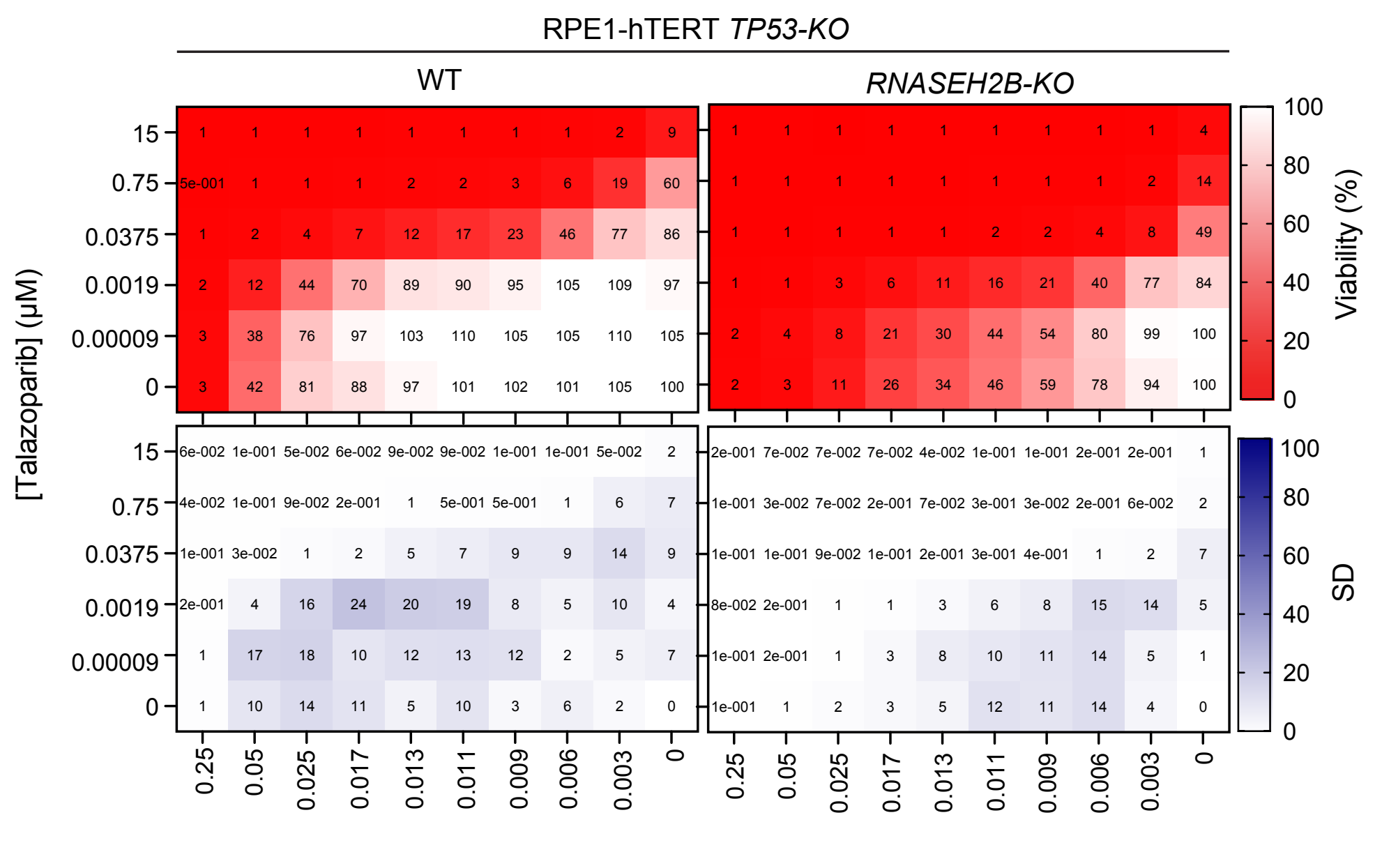

RP-3500 ( $\mu \mathrm{M})$

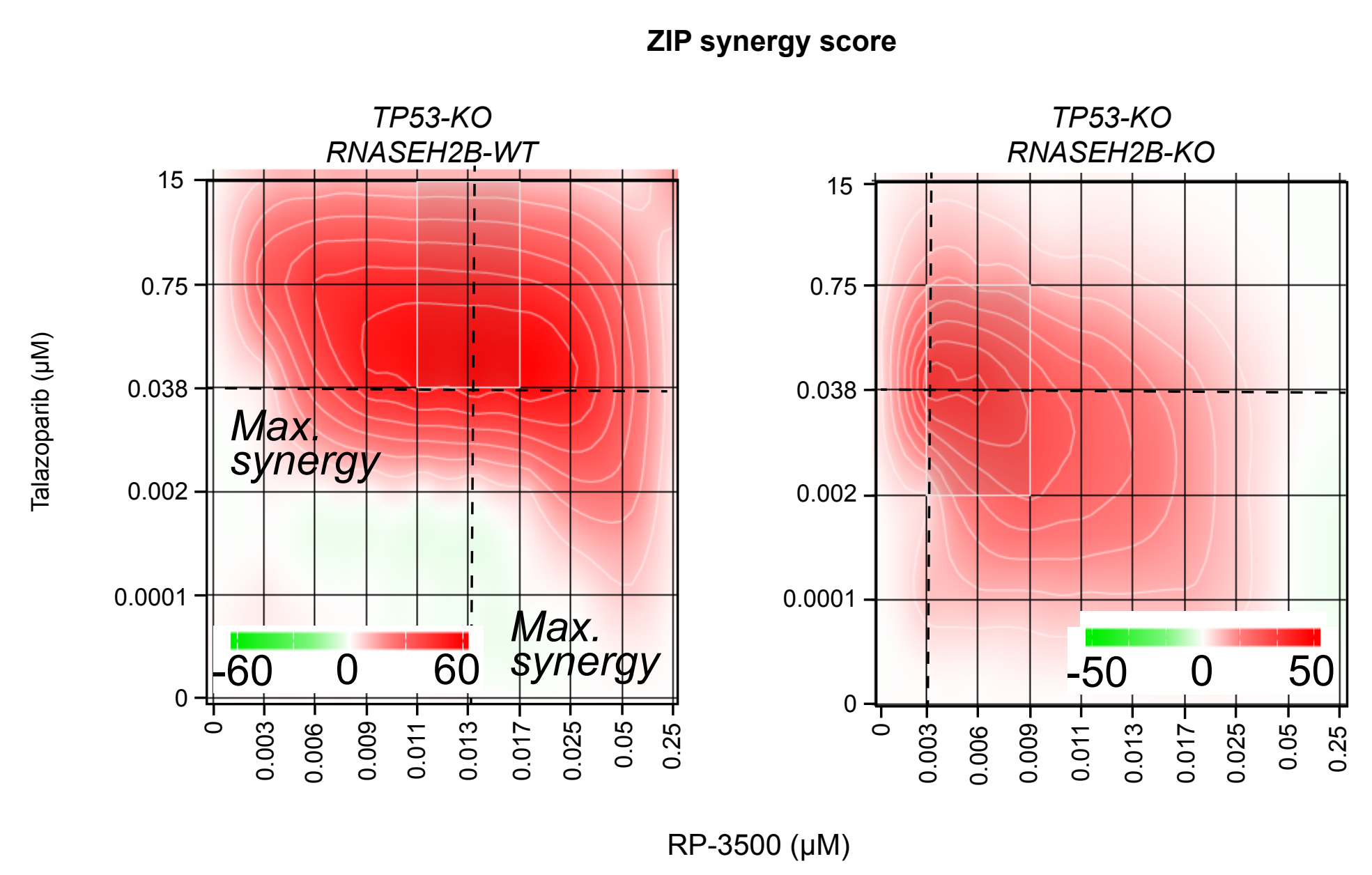


A

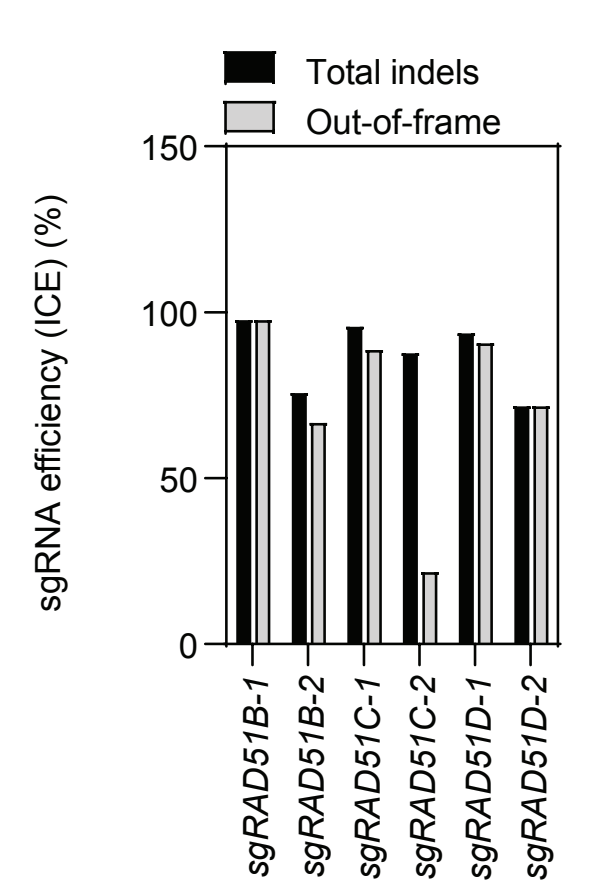

B

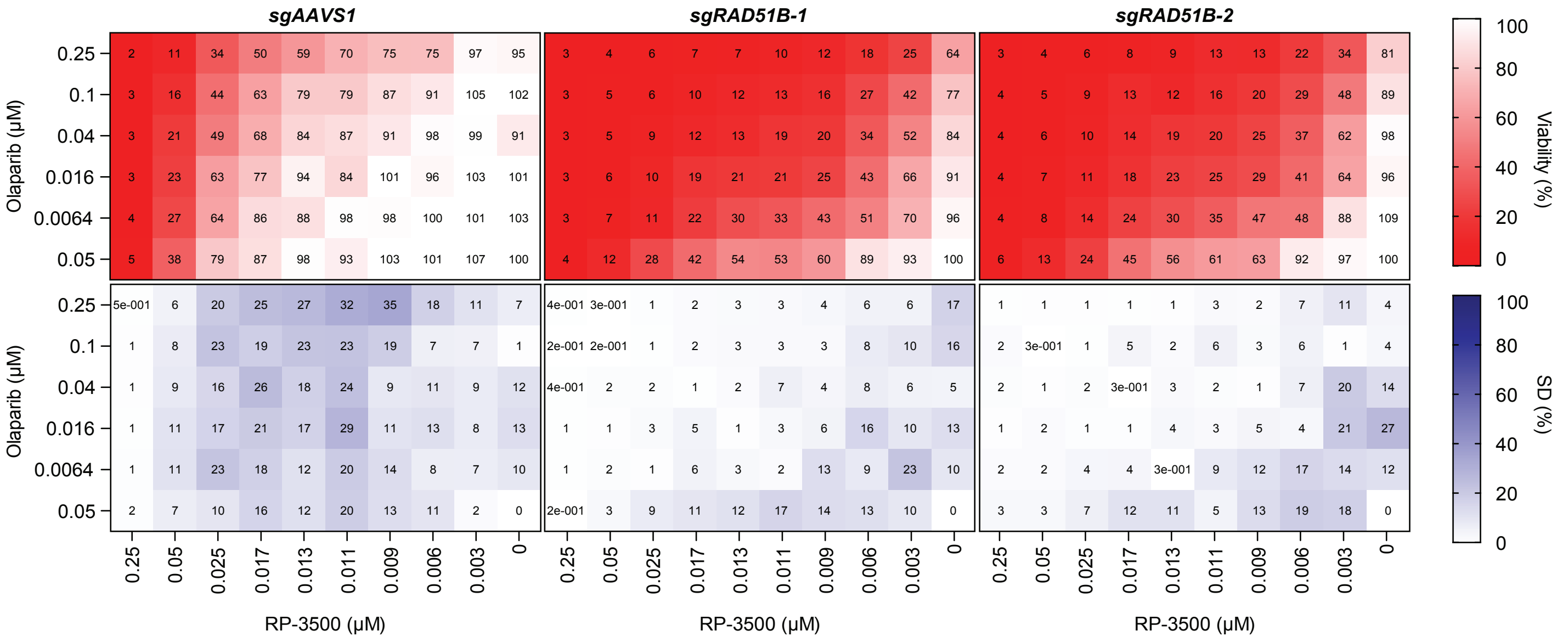

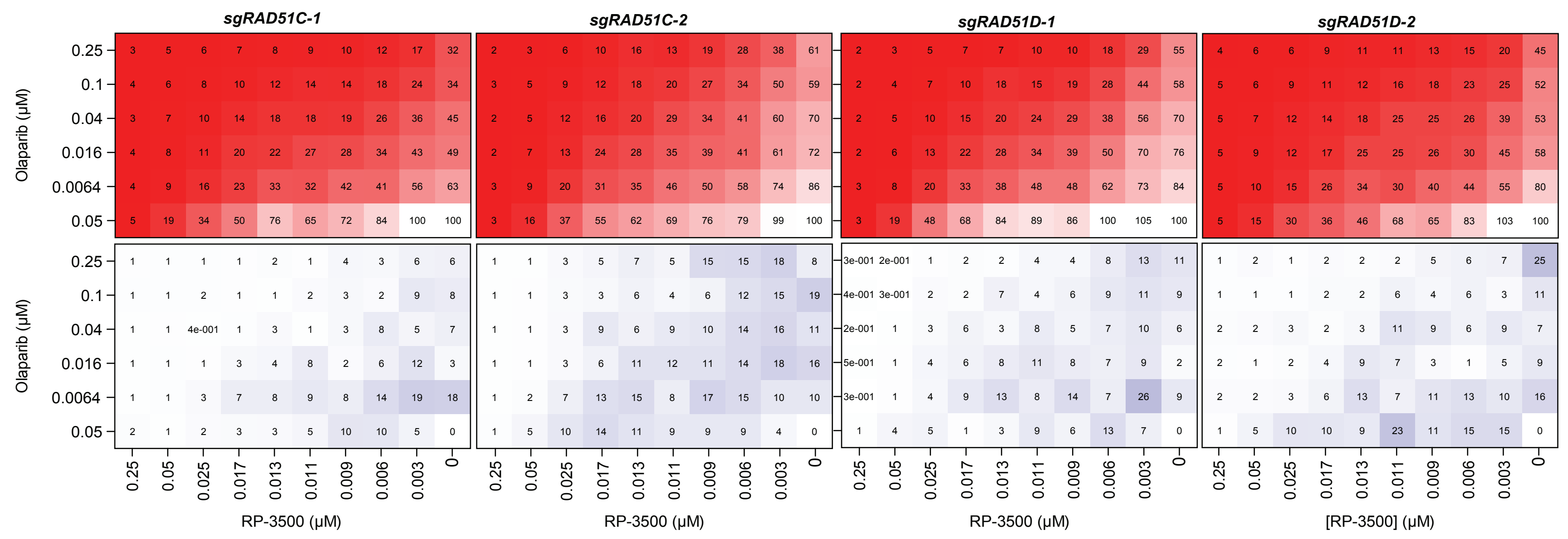


A

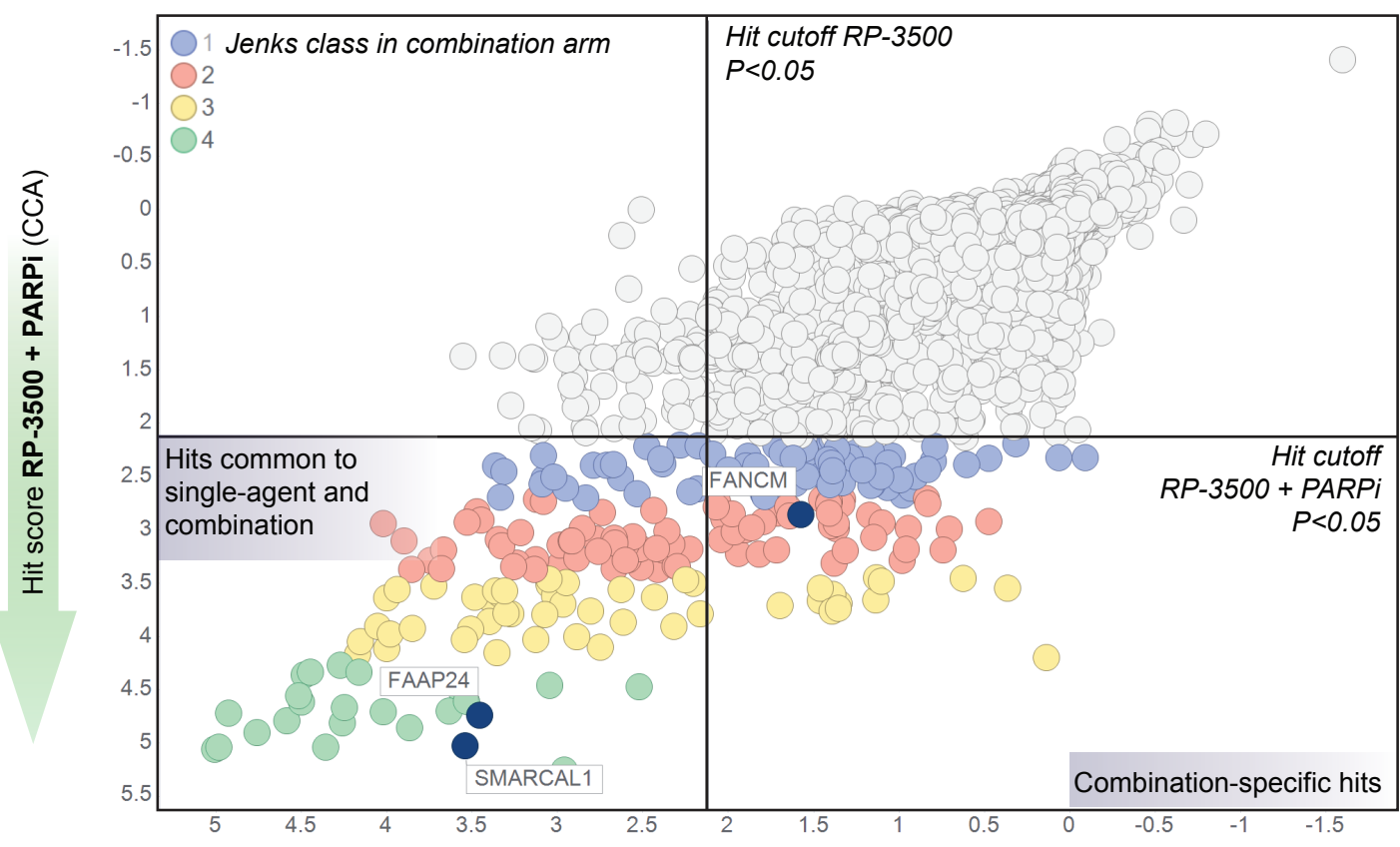

B

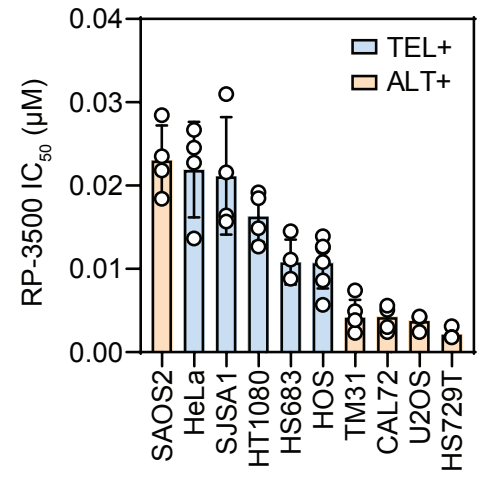


A

$72 \mathrm{~h}$ time point: 5637 WT

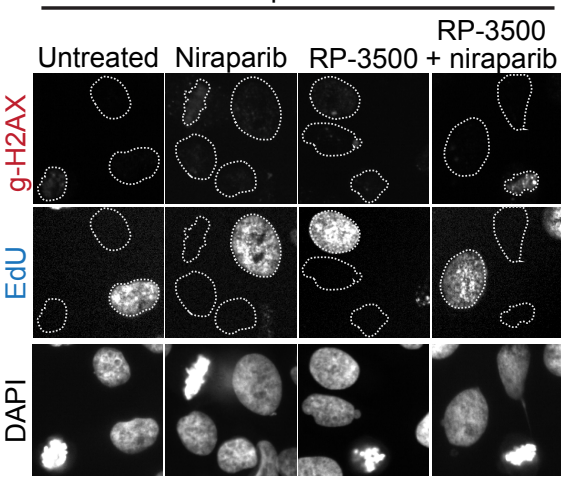

RNASEH2B-KO

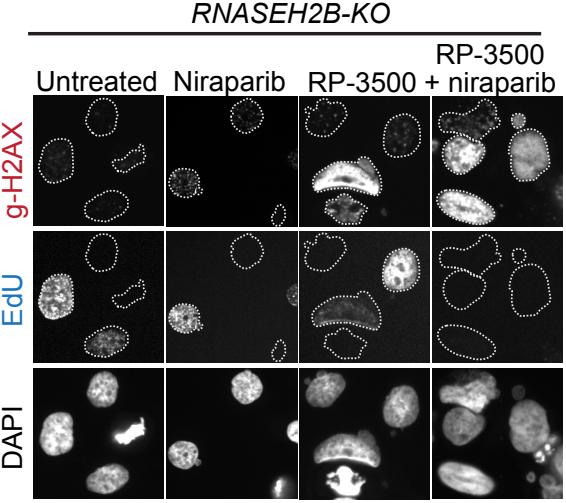

C

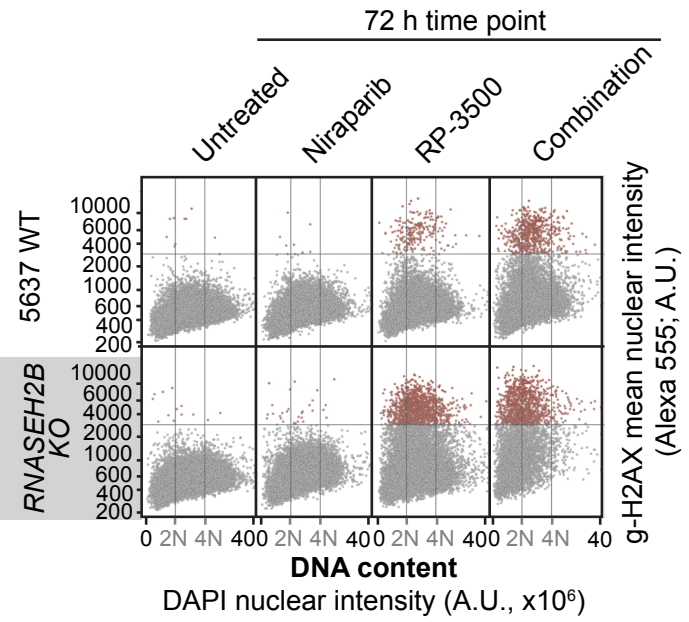

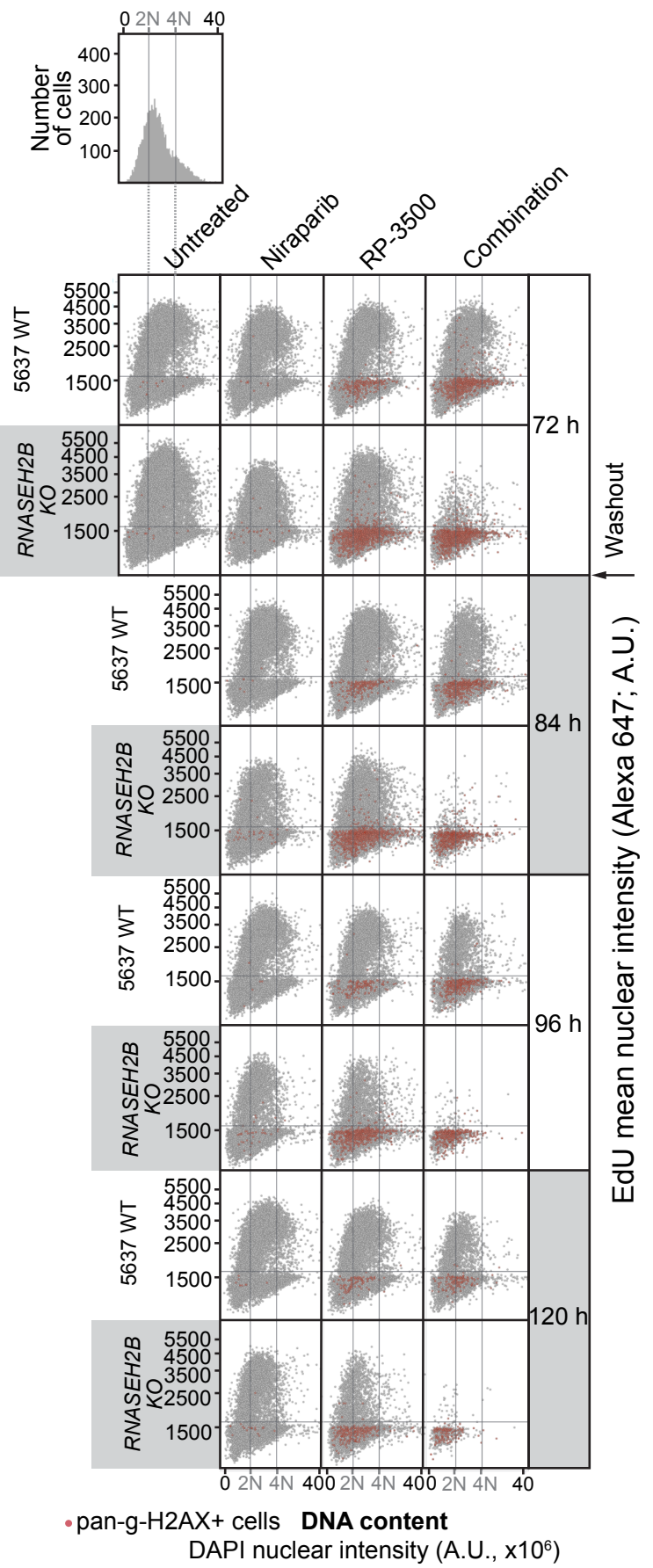


A

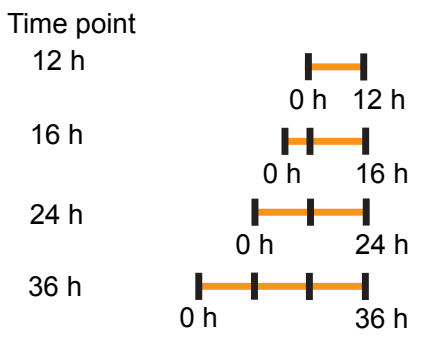

Untreated,

niraparib (PARPi, $300 \mathrm{nM})$, RP-3500 (ATRi, $10 \mathrm{nM}$ )

or

RP-3500 + niraparib

C

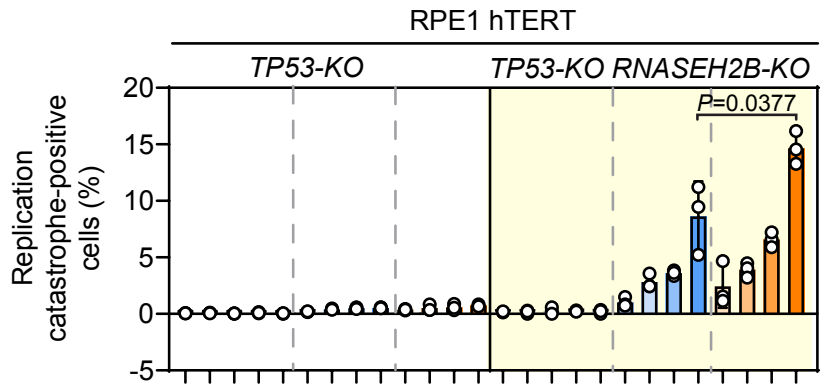

RP-3500 (10 nM) - . . . + + + + + + + . . + + + + + + +

Niraparib $(300 \mathrm{nM})+++++\ldots-\ldots+++++++++\ldots .+++++$

Time point (h) $121624361216243612162436 \quad 121624361216243612162436$

\section{E}

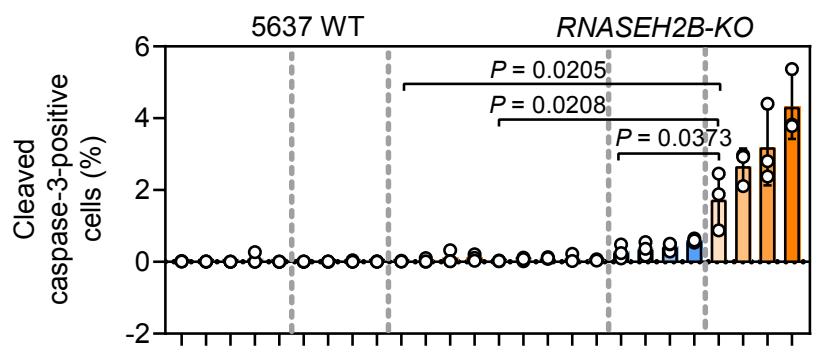

RP-3500 (8 nM) - . - . + + + + + + + - . - + + + + + + + +

Niraparib $(200 \mathrm{nM})-++++\ldots \ldots++++\ldots+++\ldots \ldots++++$

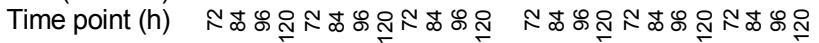

$$
\overline{\mathbf{t}_{*}} \overline{\boldsymbol{t}_{*}} \overline{\boldsymbol{t}_{*}} \overline{\mathbf{t}_{*}} \overline{\begin{array}{c}
\tau \\
\boldsymbol{t}_{*}
\end{array}}
$$
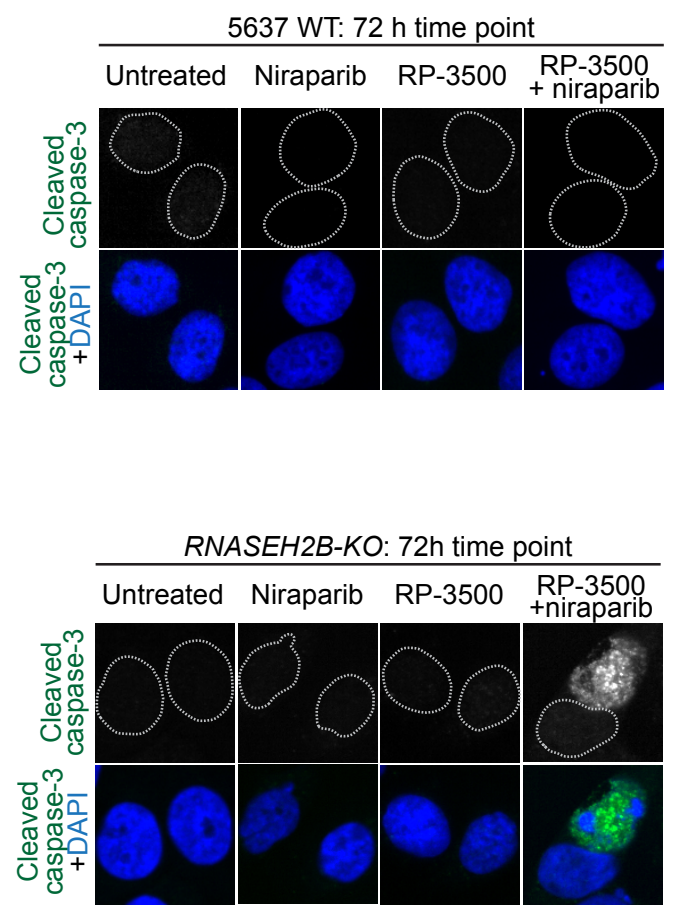
A

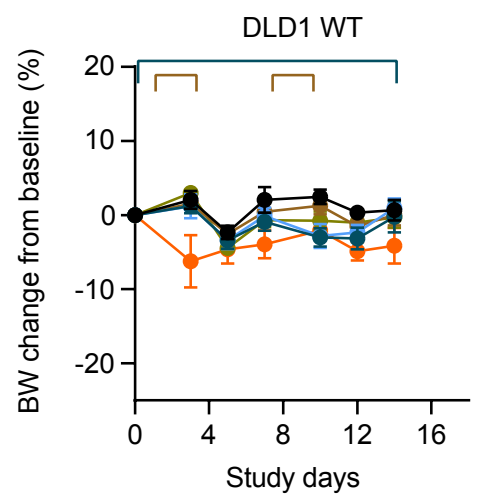

B
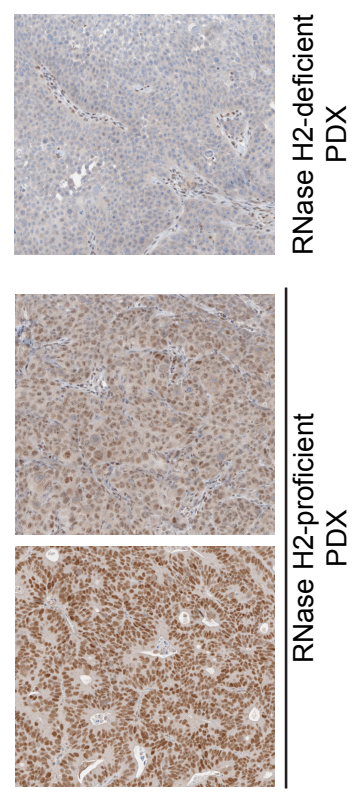

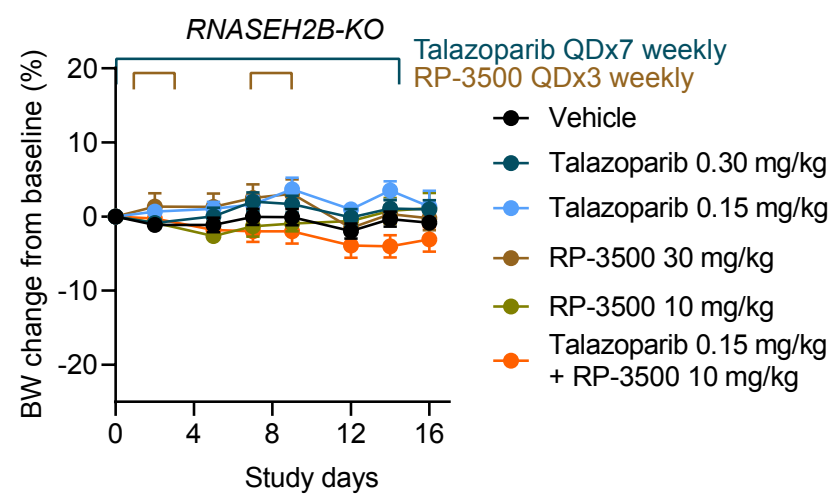

C

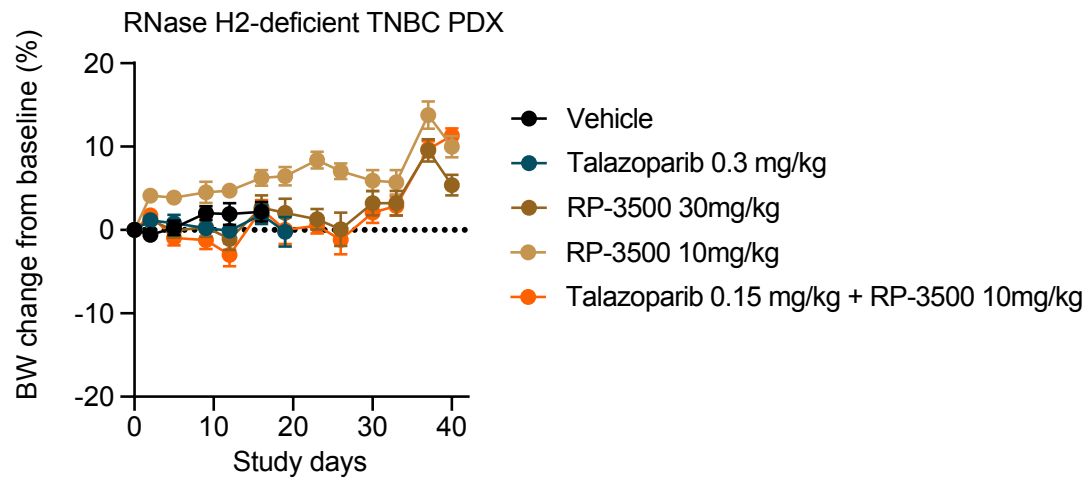

D

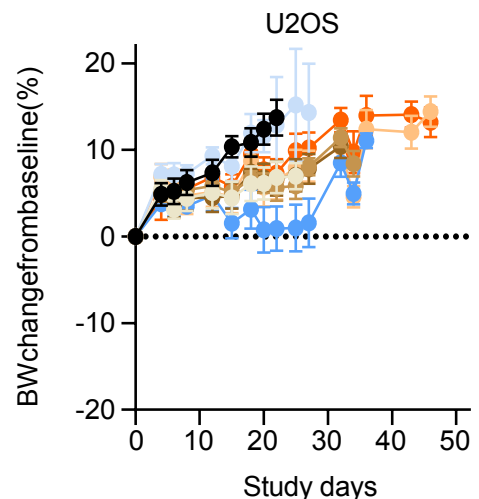

- Vehicle

RP-3500 (7mg/kg)

-- RP-3500 (15mg/kg)

- RP-3500 (30mg/kg)

- Niraparib (50mg/kg)

- Niraparib (70mg/kg) QDx5

- RP-3500 (7mg/kg) + Niraparib (50mg/kg)

- - RP-3500 (15mg/kg) + Niraparib $(50 \mathrm{mg} / \mathrm{kg})$ 